\title{
THE COMPOSITION OF HB STARS: RR LYRAE VARIABLES ${ }^{1}$
}

\author{
G.CLEMENTINI ${ }^{2}$ \\ Osservatorio Astronomico di Bologna, CP 596, I-40126 Bologna \\ E. CARRETTA \\ Dipartimento di Astronomia, Universitá di Padova, Vicolo dell'Osservatorio 5, I-35122 \\ Padova and Osservatorio Astronomico di Bologna, CP 596, I-40126 Bologna \\ R. GRATTON \\ Osservatorio Astronomico di Padova, Vicolo dell'Osservatorio 5, I-35122 Padova \\ R. MERIGHI \\ Osservatorio Astronomico di Bologna, CP 596, I-40126 Bologna \\ J. R. MOULD \\ M.Stromlo and Siding Spring Observatories, Institute of Advanced Studies, Australian \\ National University, Weston PO, ACT 2611, Australia \\ J. K. McCARTHY \\ Palomar Observatory, California Institute of Technology, Pasadena, CA 91125
}

\footnotetext{
${ }^{1}$ Based in part on data obtained with the Palomar $1.5 \mathrm{~m}$ telescope, which is owned and operated jointly by the California Institute of Technology and the Carnegie Institute of Washington, and in part on data obtained with the Asiago Astrophysical Observatory $1.8 \mathrm{~m}$ telescope

${ }^{2}$ Guest observer, Palomar Mountain Observatory
} 


\begin{abstract}
We have used moderately high-resolution, high $\mathrm{S} / \mathrm{N}$ spectra to study the chemical composition of 10 field ab-type RR Lyrae stars. Variables having accurate photometric and radial velocity data were selected, in order to derive a precise estimate of the atmospheric parameters independently of excitation and ionization equilibria. A new temperature scale was determined from literature Infrared Flux Method measures of subdwarfs and the Kurucz (1992) model atmospheres, and used to calibrate colors for both dwarfs and RR Lyraes. Photometric reddening estimates for the program stars were carefully examined, and compared with other determinations. The applicability of Kurucz (1992) model atmospheres in the analysis of RR Lyraes at minimum light was analyzed: we found that they are able to reproduce colors, excitation and ionization equilibria as well as the wings of $\mathrm{H}_{\alpha}$. The comparison solar abundances were carefully determined. From a new analysis of weak Fe I lines with accurate $g f \mathrm{~s}$ (Bard \& Kock 1994) we derived $\log \epsilon(F e)_{\odot}=7.52$, in agreement with the Fe abundances determined from meteorites and Fe II lines.

We derived abundances for 21 species. Main results are:

- The metal abundances of the program stars span the range $-2.50<[\mathrm{Fe} / \mathrm{H}]<+0.17$.

- Lines of most elements are found to form in LTE conditions. Fe lines satisfy very well the excitation and ionization equilibria. A comparison with statistical equilibrium computations shows that rather large collisional cross sections are required to reproduce observations. If these cross sections are then used in the analysis of the formation of Fe lines in subdwarfs and RGB stars, no significant departures from LTE are found for these stars, thus validating the very numerous LTE analyses.

- RR Lyraes share the typical abundance pattern of other stars of similar $[\mathrm{Fe} / \mathrm{H}]: \alpha$-elements are overabundant by $\sim 0.4$ dex and $\mathrm{Mn}$ is underabundant by $\sim 0.6$ dex in stars with $[\mathrm{Fe} / \mathrm{H}]<-1$. Solar scaled abundances are found for most of the other species, except for the low Ba abundance in the extremely metal-poor star X Ari $([\mathrm{Fe} / \mathrm{H}] \sim-2.5)$.

- Significant departures from LTE are found for a few species: Nd II, Ce II, Y II and Sc II are severely underabundant ( 0.5 dex $)$ in metal-rich variables; Ti I and Cr I are slightly $(\sim 0.1-0.2$ dex $)$ underabundant in metal-poor stars. These effects are attributed to overionization. We suggest that the photoionization of the alkaline earth-like ions is due to
\end{abstract}


Lyman lines emission produced by the shock waves that propagate in the atmosphere of these variables (Fokin 1992).

- Departures from LTE were considered in detail in the derivation of abundances for the light elements ( $\mathrm{O}$ and $\mathrm{Na}$ ). Significant corrections were required for the O I IR triplet and the Na D lines. The resulting pattern reproduces that observed in less evolved field stars. We did not find any evidence for an O-Na anti-correlation among these field HB-stars, suggesting that the environment is likely to be responsible for the anti-correlation found in metal-poor globular cluster stars (Sneden et al 1992).

We used our new $[\mathrm{Fe} / \mathrm{H}]$ abundances, as well as values from Butler and coworkers (corrected to our system), and from high resolution spectroscopy of globular clusters giants, to obtain a revised calibration of the low-resolution metallicity index $\Delta \mathrm{S}$ (Preston 1959):

$$
[\mathrm{Fe} / \mathrm{H}]=-0.194( \pm 0.011) \Delta S-0.08( \pm 0.18)
$$

Our new metallicity scale is stretched on both low and high metallicity ends with respect to Butler's (1975). The error in $[\mathrm{Fe} / \mathrm{H}]$ by $\Delta$ Sobservations is 0.16 dex, well of the same order of high resolution metallicity determinations. The slope of the calibration obtained considering only stars with $4<\Delta \mathrm{S}<10$ is slightly smaller than that obtained using all stars. While this difference is only barely significant, it might point out the presence of a non-linearity of the $\Delta \mathrm{S} v s$ $[\mathrm{Fe} / \mathrm{H}]$ relation, as suggested by Manduca (1981).

The new $[\mathrm{Fe} / \mathrm{H}]$ values were used to update the metallicity calibration of the Ca II K lineindex (Clementini et al 1991). Using the present new metallicities, and $W^{\prime}(K)$ values and relative errors from Clementini et al (1991), a least-squares fit weighted both in $W^{\prime}(K)$ and $[\mathrm{Fe} / \mathrm{H}]$ gives:

$$
[\mathrm{Fe} / \mathrm{H}]=0.65( \pm 0.17) W^{\prime}(K)-3.49( \pm 0.39)
$$

Finally, our new metallicity scale was used to revise the metallicity dependence of the absolute magnitude of RR Lyraestars, $\mathrm{M}_{V}$. Using $\mathrm{M}_{V}$ values from Fernley (1994) for the field stars, and estimates from Liu \& Janes (1990b) and Storm et al (1994) for the cluster variables, we found:

$$
\mathrm{M}_{V}=0.20( \pm 0.03)[\mathrm{Fe} / \mathrm{H}]+1.06( \pm 0.04)
$$

and:

$$
\mathrm{M}_{V}=0.19( \pm 0.03)[\mathrm{Fe} / \mathrm{H}]+0.96( \pm 0.04)
$$


the last being obtained by using $\mathrm{M}_{V}$ estimates derived for a value of the conversion factor between observed and true pulsation velocity $p=1.38$ (Fernley 1994). The adoption of the new metallicity scale does not yield significant changes in the slope and zero-point of the $\mathrm{M}_{V}$ vs $[\mathrm{Fe} / \mathrm{H}]$ relation. Observations do not rule out the possibility that the slope of the $\mathrm{M}_{V}$ vs $[\mathrm{Fe} / \mathrm{H}]$ relation might be different for metal-poor and metal-rich variables. However, a larger sample of Baade-Wesselink $\mathrm{M}_{V}$ determinations is requested to definitely settle this question.

Subject headings: Stars: variables (RR Lyrae) - Stars: abundances Stars: atmospheres - Clusters: globular - Sun: abundances 


\section{INTRODUCTION}

This is the second in a series of papers dealing with abundance analysis of RR Lyrae stars from high resolution spectroscopy (Clementini et al 1994a, hereafter Paper I). In Paper I we successfully applied our technique to derive abundances of three $a b$-type RR Lyrae variables in the globular cluster M4. Here we extend our study to a larger sample of bright field $a b$-type RR Lyraes, using high quality observational material explicitly taken for this purpose.

A number of factors make elemental abundance analysis of RR Lyrae stars an extremely worthwhile challenge:

- (a) RR Lyrae variables are powerful tools to study the chemical composition of the halo and disk of our Galaxy, and of the globular clusters. RR Lyraes have been used to derive the metal abundance of: (i) globular clusters (Smith \& Butler 1978, Smith 1984, Costar \& Smith 1988), (ii) Baade's Window (Walker \& Terndrup 1991), (iii) the Galactic Halo, and to study the metallicity distribution as a function of the galactocentric distance (Suntzeff et al 1991, hereinafter SKK91). Since these variables are generally too distant to allow a direct measure of their metallicity with high resolution spectroscopy, the most commonly used method to derive their metal abundance is via the spectrophotometric index $\Delta \mathrm{S}$ (Preston 1959). $\Delta \mathrm{S}$ measures the difference in tenths of spectral class between the spectral type of an $a b$-type RR Lyrae at minimum light estimated from the hydrogen lines, and that estimated from the Ca II K lineintensity, and it is correlated to the $[\mathrm{Fe} / \mathrm{H}]$ abundance of the star. Butler (1975) empirically derived the following metallicity calibration for $\Delta S$ :

$$
[\mathrm{Fe} / \mathrm{H}]=-0.16 \Delta S-0.23 \quad(1)
$$

using metal abundances measured from curve of growth analysis of 13 field RR Lyrae stars. (We adopt the usual spectroscopic notation, namely: $[X] \equiv \log (X)_{\text {star }}-\log (X)_{\odot}$ for any abundance quantity $X$, and $\log \varepsilon(X)=\log \left(N_{X} / N_{H}\right)+12.0$ for absolute number density abundances). Butler's calibration was further confirmed by the abundance analysis of Butler \& Deming (1979). More recently Clementini et al (1991) have used the equivalent width of the Ca II K line, $\mathrm{W}^{\prime}(\mathrm{K})$, to derive the metal abundance of field RR Lyraes. The $\mathrm{W}^{\prime}(\mathrm{K})-[\mathrm{Fe} / \mathrm{H}]$ relation $\left([\mathrm{Fe} / \mathrm{H}]=0.53 \mathrm{~W}^{\prime}(\mathrm{K})-3.08\right)$ is tighter than the one involving the $\Delta \mathrm{S}$ index; however, it is again based on the Butler (1975) and Butler \& Deming (1979) abundances. Butler's abundances, however, are now rather old and his work "needs to be redone with high $\mathrm{S} / \mathrm{N}$, high dispersion digital data, using modern synthetic spectral codes" (Suntzeff et al 1994, hereinafter SKK94). 
In spite of the large use of RR Lyraes as metallicity indicators, the literature of the last decade contains no modern redeterminations of the original metal abundances on which the $\Delta \mathrm{S}$ and $\mathrm{Ca}$ II $\mathrm{K}$ line indices are based. In this paper we present abundance analysis for 10 field RR Lyraes, 4 of which taken from Butler original sample, using high S/N (>200), moderately high resolution ( 18000) CCD spectra obtained with the Cassegrain echelle spectrograph of the Palomar 60-inch telescope (McCarthy, 1988). Our $[\mathrm{Fe} / \mathrm{H}]$ abundances are used to derive a revised calibration of the $\Delta \mathrm{S}$ and Ca II K line indices. The new metallicity scale is then compared with the globular clusters metallicity scale.

- (b) RR Lyraestars are primary distance indicators for our own and nearby galaxies because they are easily detected even at a large distance and exhibit a relatively small dispersion in their intrinsic luminosities. RR Lyraes have been detected in the Magellanic Clouds (see e.g. Walker 1991), in M31 (Pritchet \& Van den Berg 1987), and in a few galaxies of the Local Group (Saha et al 1992a,b and references therein). The absolute magnitude of RR Lyraes $\left(\mathrm{M}_{V}\right)$ is derived with a rather well established accuracy by means of the Baade-Wesselink (B-W) method (Liu \& Janes 1990a,b, Jones et al 1992, Cacciari et al 1992), which also defines the $\mathrm{M}_{V}$ vs $[\mathrm{Fe} / \mathrm{H}]$ dependence. We have used our $[\mathrm{Fe} / \mathrm{H}]$ abundances to revise the $\mathrm{M}_{V}$ vs $[\mathrm{Fe} / \mathrm{H}]$ relation.

- (c) RR Lyraes can be used to investigate the origin and evolution of the abundance anomalies found for giants in globular clusters. In a series of papers, Kraft and coworkers have found that in halo giants belonging to the field and to globular clusters there is a global anticorrelation of $[\mathrm{Na} / \mathrm{Fe}]$ and $[\mathrm{O} / \mathrm{Fe}]$ (see e.g. Sneden et al 1994). It is not clear if this anticorrelation extends to metal-rich clusters $([\mathrm{Fe} / \mathrm{H}]>-1)$, since it has not been found for giants in 47 Tuc (Brown \& Wallerstein 1992; Carretta \& Gratton 1992) and M71 (Sneden et al 1994). The most plausible interpretation for this anticorrelation is that the $\mathrm{O}$ decline is the result of deep mixing, in which some ${ }^{23} \mathrm{Na}$ produced by proton captures on ${ }^{22} \mathrm{Ne}$ is dredged up to the surface (Denisenkov \& Denisenkova 1990; Langer et al 1993). While the effect is primarily correlated to the evolutionary state, it seems to be modulated by some other mechanism, which may be meridional circulation activated by core rotation (Sweigart \& Mengel 1979). Atmospheric effects or departures from LTE are less likely to be important (Drake et al 1992, 1993). Observations of $\mathrm{Na}$ and $\mathrm{O}$ abundances in RR Lyrae stars may play an important role, since these stars are in an evolutionary phase following that observed on the giant branch. It should then be possible to observe some RR Lyrae variables which have low $\mathrm{O}$ and high $\mathrm{Na}$ abundances: this should be explored by means of accurate spectroscopic analyses, taking into account the possibility of departures from LTE. 
- (d) Quantitative estimates of statistical equilibrium in late type stars have been up to now hampered by our poor knowledge of collisional cross sections, mainly those with neutral $\mathrm{H}$ atoms; these last can only be obtained theoretically, owing to difficulties in the related experiments. Steenbock \& Holweger (1984) suggested that this mechanism plays an important thermalizing rôle in late type stars, on the basis of order-of-magnitude estimates by Drawin $(1968,1969)$. Drawin formulas after Steenbock \& Holweger have since been used in various estimates of non-LTE effects in late type stars (see e.g. Steenbock 1985). However, recently Caccin et al (1993) used more reliable estimates by Kaulakys $(1985,1986)$ to show that at least for $\mathrm{Na}$, Drawin formulas severely overestimate the cross sections for HI collisions. Given the large theoretical uncertainties, a parametric approach may be used, where collisional cross sections are estimated by matching observed non-LTE features in stars where they are expected to be large. These cross sections may then be used to predict departures from LTE in other stars, where they are expected to be smaller. Due to the combination of atmospheric parameters (gravity $g$, effective temperature $T_{\text {eff }}$ and overall metal abundance), and to the presence of shock waves in their atmospheres caused by the pulsation mechanism itself, departures from LTE are expected to be non-negligible in RR Lyraestars. Since the atmospheric parameters for abundance analysis can be determined from the light and radial velocity curves of these variables without any a priori assumption about excitation and ionization equilibria, any observed difference in abundances derived from neutral and ionized species or among lines of different excitation can be considered, reliably enough, as a hint of departures from the classical LTE assumption. Note, however, that this guess can be misguided by uncertainties existing in the knowledge of the temperature stratification for real variable stars compared to the hydrostatic equilibrium model atmospheres adopted in the present analysis. This effect may be significant owing to the dynamical character of RR Lyrae atmospheres. Hence, an estimate of the reliability of the adopted model atmosphere must be performed with appropriate tests, such as the ability to predict colors or the profiles of strong lines. Anyway, if we are able to obtain a quantitative estimate of these effects on the derived abundances, we could also put a firm upper limit on the influence of departures from LTE in the derivation of the chemical composition for stars cooler and less luminous than RR Lyraestars, e.g. red giant branch (RGB) stars and subdwarfs.

The observational material is presented in Section 2, where we describe the star selection, the data reduction procedures, the equivalent width measurements, and the radial velocities determined from the spectra. The adopted atmospheric parameters are discussed in Section 3, where emphasis is given to the determination of accurate temperatures 
using a new calibration of color indices, and to a discussion on the ability of the new model atmospheres by Kurucz (1992) to reproduce the spectra of RR Lyraes at minimum light. The results of the abundance analysis are given in Section 4; care was devoted to a comparison with solar data, and statistical equilibrium computations were performed for $\mathrm{Fe}, \mathrm{Na}$, and $\mathrm{O}$ lines. Results for all elements are also compared with those obtained for less evolved stars. Section 5 presents new calibrations of the $\Delta \mathrm{S}$ and Ca II $\mathrm{K}$ line indices; a comparison of our results with those obtained from high dispersion spectroscopy of red giants in globular clusters; and finally a discussion of the impact of our abundances in the $\mathrm{M}_{V}-[\mathrm{Fe} / \mathrm{H}]$ relation for RR Lyraes. Conclusions are summarized in Section 6.

\section{OBSERVATIONAL MATERIAL}

\subsection{Star selection and observations}

Spectra of the program stars were obtained with the Cassegrain echelle spectrograph of the Palomar 60-inch telescope during the four nights: 28 July - 1 August 1993. The P60 echelle spectrograph (McCarthy 1988) was operated in echelle grating mode using a 52.65 lines $/ \mathrm{mm}$ echelle grating and quartz prism cross-dispersers which yield a resolution $\mathrm{R}=\lambda / \Delta \lambda=38000$ per pixel, and large wavelength coverage (3400 $\AA \leq \lambda \leq 9900 \AA)$. Spectra are recorded on a TI $800 \times 800$ pixels backside illuminated CCD $(15 \mu \mathrm{m}$ pixel size $)$. The slit width was set at 1.43 arcsec, which projects to 2.1 pixels on the detector.

The FWHM measured from Th-Ar lines is $\sim 0.23 \AA$ at $4300 \AA$. The echelle grating of the P60 spectrograph has an off-plane angle $(\gamma)$ of about 10 degrees which produces a variable tilting along each order. In the data reduction phase we have neglected this effect in order to simplify the reduction procedure. This results in a mean degradation of the resolution of about $10 \%$ for stellar sources. However, in our spectra the resolution is limited to about 10000 by the large amplitude velocity fields in the atmosphere of the program variable stars. The data cover the spectral range 3400-9900 $\AA$ with 65 orders, partially overlapping for $\lambda \leq 7000 \AA$. Exposures of a Th-Ar lamp were taken with the telescope in the same position of the object to perform the wavelength calibration. Flat field and bias exposures were taken routinely at the beginning and end of each night, particularly to monitor slight temperature fluctuations of the CCD Dewar.

We took 50 spectra of 10 ab-type RR Lyraestars close to the minimum light of the variables. For one of the program star, (namely SW And), we obtained also spectra 
around the maximum light phase (see Section 4.1.5). Spectra of 5 candidate red horizontal branch stars (RHB) were also taken for the purpose of making a comparative abundance analysis. Here we will confine our study to the RR Lyrae stars; observations are in progress on a larger sample of RHB stars using the $1.8 \mathrm{~m}$ telescope of the Asiago Astrophysical Observatory. A hot bright star was observed at the beginning of each night and used during the reduction phase to locate the echelle orders within the frame.

Since one of the primary purposes of our study was to obtain a new calibration of the $\triangle \mathrm{S}$ and $\mathrm{Ca}$ II $\mathrm{K}$ line indices we included in our program some of the objects in the Butler (1975) and Clementini et al (1991) original lists. We ensured also that our program stars had known $\Delta \mathrm{S}$ and covered a large range in metallicity $(-2.2<[\mathrm{Fe} / \mathrm{H}]<0.1)$. The sample of objects ultimately observed by us at Palomar had 4 stars in common with a sample of bright field RR Lyrae variables being studied spectroscopically by Heath et al (1995). The objects in common should allow the two sets of results to be integrated once both independent abundance analyses are complete.

To make an accurate abundance analysis we need knowledge of the atmospheric parameters (gravity and effective temperature) of the stars. The gravity can be derived from the radial velocity curves of the stars while the effective temperature can be estimated from their color curves by means of a model atmosphere (see Paper I and Section 3 for a more detailed discussion of these topics). In our selection we therefore chose objects for which recent $B V R I$ and possibly $K$ light curves and accurate radial velocities were available in the literature (see references in Sections 3.2 and 3.4.1).

The currently available model atmospheres (Kurucz 1992; hereinafter K92) are stationary LTE models; however, due to pulsation, the use of a stationary model atmosphere for an RR Lyrae is an approximation not always valid. It has been found, in fact, that shock waves propagate throughout the atmospheres of these variables (Preston \& Paczynski 1964, Gillet \& Crowe 1988, Clementini et al 1994b). The presence of shocks is associated with the appearance of the bumps and humps in the light curve of the variables. Static model atmospheres are a good approximation for these stars only if the regions of the shocks are avoided. To meet these requirements, our exposures were generally shorter than 60 minutes and our spectra were all acquired near minimum light (see discussion in Section 3.4). Since most of our targets have updated ephemerides, we could safely locate the minimum light and properly phase our exposures. The list of objects is shown in Table 1 together with the adopted ephemerides for each object (Columns 4 and 5), the Heliocentric Julian Day (HJD) at half exposure of each spectrum (Column 6), and the corresponding phase (Column 7). Finally, the last column gives the references for the adopted ephemerides. 


\subsection{Data reduction}

The first part of the data reduction was performed using the facility ECHELLE in the IRAF 3 package.

We used spectra of four bright stars (one for each night) to locate the echelle orders within the frame. The position of each order was then traced interactively with a cubic spline. Assigning the bright star as a reference, order finding and tracing was performed on science frames. Scattered light (and, hence, also the bias) was then eliminated with a two-dimensional fit of the interorder regions across and along the dispersion axis. The spectra corresponding to the recorded echelle orders were extracted from each of these cleaned frames.

Two-dimensional dispersion solutions were found for the Th-Ar arc spectra. The typical r.m.s. deviation of the arc lines from the fitted wavelength calibration polynomial was $\sim 0.02 \AA$. This can be regarded as the internal accuracy in our wavelength calibration. The reduction of the arc spectra confirmed the spectrograph stability during multiple observations of the same object. Out of 65 orders found, we retained for further analysis only those 52 that cover the spectral region between 3790 and $9160 \AA$, because $\mathrm{S} / \mathrm{N}$ is low outside this range. Further reduction and data analysis were carried out using the ISA (Gratton 1988) package, purposely designed for one dimensional high resolution spectra. We found that division by lamp flats did not yield satisfactory results at long wavelengths due to the presence of rather strong interference fringes which were not well divided out with this technique. Better results were achieved by dividing the spectra by a pseudo-flat field obtained from the spectrum of X Ari, the most metal-poor star in our sample, for which a fiducial continuum may be easily identified by means of cubic spline interpolation through selected spectral points. This technique allowed us to properly correct interference fringes up to $\lambda<7300 \AA$. Beyond this limit the only spectral feature we examined was the permitted O I triplet at 7771-7774 $\AA$ (see Section 4.5). Single spectra of each star were compared to eliminate the (very few) cosmic ray spikes, and then summed. Spectra were not shifted before adding them together to account for differences in radial velocity because the effect of the variation in radial velocity at the observed phases is negligible at our spectral resolution. Giving the brightness of the program stars and the small size of the slit, sky contamination can be safely neglected: therefore no sky subtraction was performed on the spectra. Whenever relevant (5860-5920, 6270-6320 and 7080-7130 $\AA$ ),

\footnotetext{
${ }^{3}$ IRAF is distributed by the National Optical Astronomy Observatories, which is operated by the Association of Universities for Research in Astronomy, Inc., under cooperative agreement with the National Science Foundation.
} 
telluric lines were divided out still using the spectrum of X Ari; however, regions including strong telluric bands were not used in the analysis. In the case of the D lines region, particular care was taken to prevent the stellar and interstellar absorption lines present in the spectrum of X Ari from producing false emission features on the spectra of the other stars. For this purpose, we used also the spectra of other metal-poor stars, having different radial velocities. Finally, we traced the continuum on the co-added spectra by using a cubic spline interpolation through a few selected spectral points. Comparison with synthetic spectra showed that the final fiducial continuum was correct within $0.5 \%$, at wavelengths longer than $4500 \AA$, with the exception of the region of the Balmer lines where errors may be as large as 1-2\%. In Table 2 we list total exposures and $\mathrm{S} / \mathrm{N}$ of the co-added spectrum for each star. Small portions of the co-added normalized spectra of X Ari, RR Cet and SW And are shown in Figure 1.

\subsection{Equivalent widths}

Equivalent widths $(E W \mathrm{~s})$ for $\sim 100$ lines in the average spectrum of each star were measured by means of a gaussian fitting routine. This procedure works quite well for RR Lyrae stars because damping wings are weak for all measured lines; in a few cases, comparisons with synthetic spectra were also done. The complete list of lines and adopted gf values and their measured $E W \mathrm{~s}$ is given in Table 3a,b, and is available on electronic form from R. Gratton. Errors in these $E W$ s are mainly due to uncertainties in the location of the fiducial continuum: for this reason, $E W$ s were not measured at wavelengths shortward of $4500 \AA$ in the line rich spectra of SW And and V445 Oph, where continuum location is likely to be underestimated. Errors in the $E W$ s are not easy to accurately estimate because the measured $E W$ s should depend on phase and $S / N$ level. A rather realistic estimate can be obtained by comparing the $E W$ s measured for the same lines in the spectra of RR Lyr and RR Cet, which have similar atmospheric parameters at the average observed phases (RR Cet has only a slightly larger value of the microturbulent velocity). This comparison is shown in Figure 2, where we have overimposed the best-fit regression line with zero constant value: $E W_{\mathrm{RR} \text { Cet }}=1.052( \pm 0.008) E W_{\mathrm{RR} \text { Lyr }}$ from 76 lines; the regression coefficient is slightly different from 1 , mainly due to the different values of the microturbulent velocity. The scatter around this best-fit line is $9.3 \mathrm{~m} \AA$, if we attribute equal errors to the measure of $E W \mathrm{~s}$ in each star, we obtain an r.m.s. error of $6.6 \mathrm{~m} \AA$. Figure 2 suggests that this error is fairly independent of the $E W$ itself: hence, $10 \mathrm{~m} \AA$ is a reasonable lower limit for reliable estimates of the $E W \mathrm{~s}$. RR Cet and RR Lyr spectra have the highest $S / N$; errors in $E W \mathrm{~s}$, 
$\sigma(E W)$, for the other stars likely scale as the inverse of $S / N: \sigma(E W) \sim 2400 /(S / N) \mathrm{m} \AA$. The values of $\sigma(E W)$ for each star are given in Column 6 of Table 2.

No direct comparison of our EWs with those measured by Butler (1975) and Butler \& Deming (1979) is possible, since the spectra used in those analyses were taken close to maximum light.

Carney \& Jones (1983) analyzed photographic echelle spectra of VY Ser; their mean phase of observation is 0.615 , compared to our mean value of 0.72 . On our scales (see below), $T_{\text {eff }}$ and gravity at Carney \& Jones phase were $T_{\text {eff }}=5969 \mathrm{~K}$ and $\log g=2.60$ (their adopted values are $T_{\text {eff }}=6000 \mathrm{~K}$ and $\log g=2.3$ ), compared to the mean values for our observations of $T_{\text {eff }}=5993 \mathrm{~K}$ and $\log g=2.69$. These sets of values are very similar, so that

no important modifications of the $E W \mathrm{~s}$ are expected. A line-to-line comparison of the $E W \mathrm{~s}$ indicates that our values are smaller by $7 \pm 4 \mathrm{~m} \AA$ ( $\sigma=16 \mathrm{~m} \AA$ : 15 lines). This difference is small, and a correction of this entity would not affect significantly our analysis; however, we can expect that our $E W \mathrm{~s}$ are more reliable since our CCD echelle spectra have much higher $S / N$.

\subsection{Radial velocities and phases}

Radial velocities were measured from the average spectra using a rather large number of lines $(\sim 100)$ for each star. They are listed in Column 4 of Table 2 . These values can be used to check the phases of our spectra. Since no radial velocity standard was observed, the zero-point of these radial velocities was determined using the telluric [OI] line at 5577.341 $\AA$. Internal errors of these radial velocities, as deduced from the line-to-line scatter (reduced by the square root of the number of lines), are $\pm 0.3 \mathrm{~km} \mathrm{~s}^{-1}$; star-to-star variations in the radial velocity for the telluric line are similar $\left(\sigma=0.2 \mathrm{~km} \mathrm{~s}^{-1}\right)$, showing that spectrograph flexures are small. However, uncertainties in our radial velocities are larger, since errors in the determination of the radial velocity from individual stellar lines are as large as $2.7 \mathrm{~km} \mathrm{~s}^{-1}$, where similar contributions are due to photon noise, residual blending effects, and errors in the wavelength calibration; the last source of error (amounting to $\sim 0.02 \AA$, i.e. $\sim 1.1 \mathrm{~km} \mathrm{~s}^{-1}$ ) is systematic, and then apply also to the telluric [OI] line. On the other hand, we think that our zero-point error is not very large, as confirmed by the comparison with the expected radial velocities at the observed phases (Column 5 of Table 2) for those stars for which they may be considered reliable; there are 7 such variables (no radial velocity curve is available for VX Her; and ST Boo and RR Lyr radial velocities are more uncertain because these stars are affected by the Blazhko effect). The mean difference 
between observed and expected velocities is $V_{r}($ obs $)-V_{r}(\exp )=-0.4 \pm 0.9 \mathrm{~km} \mathrm{~s}^{-1}$. This test confirms that our ephemerides are reliable. We think that the Blazhko effect, more than errors in the adopted ephemerides, might be responsible for the higher discrepancy found for RR Lyr and ST Boo. We discuss in Section 3.4 the effect on the atmospheric parameters adopted for ST Boo and RR Lyr if phases inferred from the radial velocity measures are used in place of phases derived from ephemerides.

Finally, our spectra can be used to test the presence of systematic variations with optical depth, in radial velocities at minimum phase. This problem is of some relevance in the determination of the absolute magnitude of RR Lyrae variables with the B-W method, where the assumption is made that no velocity gradients exist in the region of formation of the lines used to measure the radial velocity (basically weak metal lines), and between this region and the continuum forming region. Jones (1987) and Clementini et al (1994b) measured the velocity of lines at different depths of formation in a sample of field and cluster RR Lyraes and reached the conclusion that no significant velocity gradients $\left(\leq 2 \mathrm{~km} \mathrm{~s}^{-1}\right)$ exist throughout the atmospheric layers where these lines are formed. Here we can repeat their test in a more stringent way. Since the stronger lines measured on our spectra (with $E W \sim 300 \mathrm{~m} \AA)$ form at much shallow optical depths $\left(\log \tau_{\text {Ross }} \sim-3\right)$ than very weak lines (forming at $\log \tau_{\text {Ross }} \sim-1$ ) t, we have checked whether a correlation exists between $E W$ and $V_{\text {rad }}$. The mean value for the linear regression coefficient $a$ in the $E W-V_{\text {rad }}$ plane over all the stars was $a=-2.3 \pm 1.9 \mathrm{~km} \mathrm{~s}^{-1} / \AA$ (where the error bar is the standard deviation of the mean over all 10 program stars). For comparison, in the same observing run we obtained spectra for a sample of non-variable metal-poor giants with $4600 \leq T_{\text {eff }} \leq 6000 \mathrm{~K}$ : the average value of $a$ for this sample was $a=0.2 \pm 1.4 \mathrm{~km} \mathrm{~s}^{-1} / \AA$. The trend of radial velocity with $E W$ s measured in RR Lyrae variables is only barely significant; we consider it as a marginal indication of a small acceleration of the outward motion throughout the atmosphere at the observed phase (i.e. at minimum).

\section{ATMOSPHERIC PARAMETERS}

As we have anticipated in Section 2.1, to perform abundance analysis we need the effective temperature, the surface gravity, the overall metal abundance and the microturbulent velocity of our program stars. Effective temperatures corresponding to the phases of our

\footnotetext{
4 We verified this assertion by computing the average depth of formation (at line center) of lines of different strength and excitation following the precepts of Magain (1986).
} 
spectra were derived from the $B-V, V-R, V-I$, and $V-K$ colors of the stars using K92 models of appropriate metallicity and gravity. Colors have been previously corrected for reddening. The procedure used to estimate the reddening is described in detail in Section 3.1. In Sections 3.2 and 3.3 we discuss the derivation of surface gravity and overall metal abundance. In Section 3.4 we describe our procedure to derive effective temperatures from K92 model atmospheres. Finally, in Section 3.5 we discuss the adopted microturbulent velocities.

\subsection{Reddening}

Reddening is a crucial point in our analysis since an error of 0.01 mag in the adopted reddening translates into an error of $\sim 50 \mathrm{~K}$ in the derived temperature; this, in turn, translates into an uncertainty in the derived abundance that for instance in the case of Fe I corresponds to $\sim 0.05$ dex. Reddening estimates for the program stars based on photometric indicators have been published by several authors (Sturch 1966, Jones 1973, Lub 1977a,b, 1979, Liu \& Janes 1990a, Blanco 1992 and reference therein). Blanco (1992) (hereinafter B92) presents a critical re-evaluation of the reddening of ab-type RR Lyraestars as derived from photometric indices. He uses a revised version of Sturch (1966) method to evaluate $E(B-V)$ from the observed near-minimum light colors for $a b$-type RR Lyraes, and derives a formula which gives $E(B-V)$ as a function of the metallicity of the star as inferred from the $\Delta \mathrm{S}$ index, the period, and the $B-V$ color during the phase interval $0.5<\phi<0.8$ :

$$
E(B-V)=<B-V>_{0.5<\phi<0.8}+0.0122 \Delta S-0.00045(\Delta S)^{2}-0.185 P-0.356
$$

Equation (2) was established using: (i) mean colors at minimum light taken, when available, from B-W analysis light curves (this is also the photometry used in the present study to derive temperatures); and (ii) $\Delta$ S values collected from the literature and reduced to Butler's equivalent values.

B92 makes a very extensive comparison with previous reddening determinations, particularly with those by Lub (1977a,b,1979), and Sturch (1966), which are found to be in good agreement with his results within an accuracy of \pm 0.02 mag. A completely independent method to estimate reddening is provided by Burstein \& Heiles (1978, 1982, hereinafter BH78 and BH82) who used H I column densities plus galaxy counts to determine the average reddening as a function of Galactic latitude and longitude, and give values appropriate for the RR Lyraes (taking into account their distances). In Table 4 we compare B92 reddening values for our stars with BH78 and BH82. Also listed are reddening values 
used in the B-W analysis of the various stars. There is generally good agreement between B92, BH78 and BH82 with the exception of BH82 values for V445 Oph and RR Lyr which deviate by a very large amount from both Blanco's and BH78 estimates, and that therefore were not considered (see also the discussion on the reddening of V445 Oph in Fernley et al 1990). In conclusion, B92 reddening estimates were adopted for our stars with the exception of V445 Oph for which a reddening value of $0.27 \mathrm{mag}$ was found to be more appropriate during the abundance analysis procedure; and of UU Cet, for which a lower metallicity compared to B92 was recently found with $\Delta$ S analysis by SKK94 (see discussion in Section 3.3). $E(B-V)$ for this star was derived from eq. (2), taking into account the new metallicity estimate. The adopted reddening values are listed in Column 2 of Table 5 . We associate with these estimates an uncertainty of $\pm 0.02 \mathrm{mag}$.

We may compare the adopted photometric estimates of reddening with those derived from interstellar absorption features present in our spectra. We measured the $E W \mathrm{~s}$ for the Diffuse Interstellar Band (DIB) at $5780 \AA$, and for the interstellar Na D lines. DIB $E W \mathrm{~s}$ were transformed into reddening by means of the calibration drawn by Herbig (1993), using only those stars (72) with $E(B-V) \leq 0.4$, matching the expected range of reddening values for the program RR Lyrae variables. The adopted calibration was $E(B-V)=(1.54 \pm 0.10) E W_{5780}$, where the $E W$ of the DIB is in $\AA$. For the Na D lines, we transformed Na I column densities deduced by means of the doublet ratio method into reddening using the relation: $E(B-V)=(3.27 \pm 0.19) 10^{-14} n(\mathrm{Na} \mathrm{I})$, obtained by Benetti et al (1994) from a compilation of literature data about interstellar lines and reddenings. Table 5 lists the relevant data. The agreement between the different estimates of the interstellar reddening is good for all stars, excluding X Ari. The Na D lines give slightly larger reddening estimates, but the difference is not significant. Mean differences are:

$$
\begin{gathered}
E(B-V)_{\mathrm{Phot}}-E(B-V)_{5780}=0.00 \pm 0.02 \mathrm{mag}, \sigma=0.06 \mathrm{mag} \\
E(B-V)_{\mathrm{Phot}}-E(B-V)_{\mathrm{Na} \mathrm{D}}=-0.03 \pm 0.02 \mathrm{mag}, \sigma=0.04 \mathrm{mag} \\
E(B-V)_{5780}-E(B-V)_{\mathrm{Na} \mathrm{D}}=-0.02 \pm 0.04 \mathrm{mag}, \sigma=0.10 \mathrm{mag}
\end{gathered}
$$

If we average the two spectroscopic determinations, the mean difference with the photometric reddening (again excluding $\mathrm{X}$ Ari) is:

$$
E(B-V)_{\mathrm{Phot}}-E(B-V)_{\mathrm{Spec}}=-0.01 \pm 0.01 \mathrm{mag}, \sigma=0.03 \mathrm{mag}
$$

While this agreement may be fortuitous (given the large spread usually existing in reddening estimates from these spectroscopic features), on the whole it supports the reddening scale adopted in this paper. 
Reddening in the $(V-R),(V-I)$ and $(V-K)$ colors was obtained from the $E(B-V)$ values in Column 2 of Table 5 using Cardelli et al (1989) absorption coefficients: $\mathrm{A}(R) / \mathrm{A}(V)=0.751, \mathrm{~A}(I) / \mathrm{A}(V)=0.479$ and $\mathrm{A}(K) / \mathrm{A}(V)=0.114$, which are valid for a standard value of the total to selective absorption $\mathrm{R}_{V}=\mathrm{A}(V) / E(B-V)=3.1$. These coefficients are very similar to those derived from Howarth (1983) formula. Cardelli et al (1989) coefficients are calculated for the Johnson photometric system. The $V-R$ and $V-I$ colors used in the present paper are in the Cousins system. Transformation between the two photometric systems is achieved using Bessell (1979) equations: $(V-R)_{C}=0.713(V-R)_{J}$ and $(V-I)_{C}=0.778(V-I)_{J}$, that lead to the following reddening corrections to apply to the observed colors: $E(V-R)_{C}=0.550 E(B-V), E(V-I)_{C}=1.257 E(B-V)$, and $E(V-K)=2.747 E(B-V)$. The use of Howarth (1983) coefficients would give reddening corrections in good agreement with those used here even for the most reddened stars, for which we would find differences $\leq 0.02$ mag.

\subsection{Gravities}

Gravity is an input parameter for abundance analysis and it is also needed to select the appropriate K92 model to derive the color-temperature transformations. Due to pulsation the gravity of an RR Lyraestar varies during the cycle. The gravity to consider is therefore the effective gravity that is described by the formula:

$$
g=G M / R^{2}+d^{2} R / d^{2} t
$$

where $\mathrm{M}$ and $\mathrm{R}$ are the mass and the radius of the star in solar units. The first term in eq. (3) is the mean gravity of the star (i.e. the gravity that the star would have if it were not pulsating), and may be derived from its mass and mean radius; the second term represents the variation of the gravity along the pulsation cycle due to the acceleration of the moving atmosphere and can be estimated by differentiating the radial velocity curve of the variable.

Eight of the objects in our list have recently been the subject of B-W analysis (Liu \& Janes 1990a, Cacciari et al 1989a,b, Cacciari et al 1992, Manduca et al 1981, Siegel 1982, Jones et al 1987, 1988, Fernley et al 1989, 1990). We have used the masses and radii estimated for them with the B-W method to derive the mean gravity term of eq. (3). Radial velocity curves are available for all the variables in our list except VX Her (Liu \& Janes 1989, Cacciari et al 1987, Clementini et al 1990, Sanford 1949, Carney \& Latham 1984, Jones et al 1987, Oke 1966, Preston \& Paczynski 1964, Clementini et al 1995), and have been differentiated to derive the acceleration term in eq. (3). For ST Boo, which does not 
have $R$ and $M$ estimates, we have assumed a mean gravity $\log G M / R^{2}=2.79$ and derived from eq. (3) $\log g=2.71$ as mean value corresponding to our spectra, in good agreement with what found for stars of similar metallicity. Lub (1977b) published $<\log g>$ values for RR Lyraestars estimated from Walraven photometric indices. He noticed that a zero-point error may be present in his gravity calibration. Indeed we found that for the stars we have in common our mean gravities are systematically lower than Lub's by $-0.28 \pm 0.09$ dex. The gravity of VX Her was then obtained from Lub (1977b) converted to our gravity scale and further lowered by 0.07 dex to take into account that our spectra are taken at minimum light. Mean effective gravities corresponding to our average spectra are listed in Column 3 of Table 10. Given the uncertainties in the adopted masses and radii involved in the above procedure, we assign to our $\log g$ estimates a conservative error of 0.20 dex.

\subsection{Metal abundance}

In the literature we found a very large collection of metallicity estimates for all the program stars. They include: $\Delta$ S estimates (Preston 1959, Butler 1975, McDonald 1979, Clube et al 1969, Alania 1973, Woolley \& Savage 1971, Smith 1990, Kinman \& Carretta 1991, SKK91, SKK94), Ca II K line estimates (Clementini et al 1991), and metallicities from abundance analysis by Butler (1975), Butler \& Deming (1979) and Carney \& Jones (1983). B92 made an extensive review of the various $\Delta \mathrm{S}$ values present in the literature which he reduced to a uniform system and averaged for the stars with independent determinations. In Table 6 we have collected the $[\mathrm{Fe} / \mathrm{H}]$ values from:

- Columns 2, 3 and 4: high resolution spectroscopy (Butler 1975; Butler \& Deming 1979; Carney \& Jones 1983);

- Column 5: Ca II K line index (Clementini et al 1991);

- Columns 6 and 7: $\Delta$ S parameter (SKK94; B92);

- Column 8: the input values used in the B-W analysis;

- Column 9: the input values used in the determination of $T_{\text {eff }}$ (see below).

In general the various estimates agree within 0.2 dex. UU Cet and V445 Oph deserve a more extended comment. SKK94 find for UU Cet a rather low metal abundance compared to other estimates. Since we do not have any reason for preferring one estimate to the others, we have used the average among the available values for this star: $[\mathrm{Fe} / \mathrm{H}]=-1.2$. 
As it will be discussed in detail in Section 3.4, a metallicity $[\mathrm{Fe} / \mathrm{H}] \sim+0.2$ was found more appropriate for V445 Oph during the abundance analysis.

With the exception of V445 Oph the values in Column 9 agree within \pm 0.10 dex with the mean of the other $[\mathrm{Fe} / \mathrm{H}]$ abundances listed in Table 6 . A variation of 0.2 dex in the input metallicity has only a marginal influence on the derived temperatures (see Section 3.4) and on the derived abundances (see Section 4)

\subsection{K92 models and the color-temperature transformations}

\subsubsection{Intrinsic colors for the program stars}

Accurate $B V R I$ photometry is available for all the stars in our sample and $K$ light curves are available for 6 of them. The observed colors we used are on the Johnson-Cousins system $\left(B V R_{C} I_{C} K_{J}\right)$. They are taken from the published works of Liu \& Janes (1989), Fitch et al (1966), Siegel (1982), Manduca et al (1981), Carney \& Latham (1984), Jones et al (1987, 1988), Fernley et al (1989, 1990), Burchi et al (1993), Clementini et al (1990, 1995), Cacciari et al (1987, 1992), Barnes et al (1988), Stepien (1972), and Sturch (1966). Original photometries were reduced to the Johnson-Cousins system using transformation equations given by Bessel $(1979,1983)$ and Jones et al (1987). A detailed description of the photometric data used in the present analysis and of the procedure used to transform original photometries to a uniform photometric system is given in the Appendix.

Data have then been re-phased according to the ephemerides in Table 1, and smoothed curves were drawn through them. Colors read from the smoothed color curves at phases corresponding to those of our spectra were corrected for reddening according to the precepts given in Section 3.1.

\subsubsection{The color-temperature transformations}

\footnotetext{
${ }^{5}$ In fact, while we found that important electron donors like $\mathrm{Mg}$ and $\mathrm{Si}$ are overabundant with respect to Fe in the program stars, the effects of variations of the overall metal abundance by a few tenths of a dex on the atmospheric structure and deduced abundance are very small, because a large fraction of the free electrons is provided by $\mathrm{H}$ in metal-poor RR Lyraestars, as verified by appropriate computations.
} 
Effective temperatures corresponding to the phases of our spectra were derived from the dereddened colors of the program stars using new color- $T_{\text {eff }}$ calibrations we explicitly determined for this purpose. They were obtained using a procedure similar to that recently followed by King (1993). This consists of two steps: first, an empirical color- $T_{\text {eff }}$ calibration is determined for population I dwarfs, based on $T_{\text {eff }}$ derived by means of the Infrared Flux (IF) method (Blackwell \& Shallis 1977). Second, the appropriate calibration for RR Lyraestars is obtained by correcting the calibration obtained for population I dwarfs by the corresponding offsets, due to differences in gravities and metal abundances, given by K92 model atmospheres. The underlying assumption is that while zero point errors may be present in the K92 theoretical colors, these model atmospheres well predict the variations of colors with gravity and metal abundances.

\section{The color- $T_{\text {eff }}$ transformations for population I dwarfs}

Fernley (1989, hereinafter F89) compared observed $V-K$ colors and effective temperatures $T_{\text {eff S }}$ derived mainly with the IF method (Saxner \& Hammarbäck 1985, hereinafter SH85) of population I main sequence stars with synthetic $V-K$ and $T_{\text {eff }}$ derived from Kurucz (1979, hereinafter K79) models, and found that theoretical and empirical $(V-K)-T_{\text {eff }}$ relations have different slopes. We have repeated Fernley's procedure on K92 models. An approach similar to ours has recently been followed by King (1993); however, this last author used the effective temperatures derived from the IF method (SH85) based on the MARCS model atmosphere by Gustafsson et al (1975). A slightly different temperature is derived using the IF method and K92 models. Blackwell \& Lynas-Gray (1994; hereinafter BLG94) published effective temperatures derived with the IF method and the new K92 model atmospheres for a large sample of bright solar metallicity stars. They also made a comparison with results from IF method and K79 models and give in their Table 2 the appropriate temperature corrections. Our procedure was the following: a list of Population I main sequence stars of spectral type A-F-G, luminosity class IV-V and with well determined effective temperatures was obtained by merging SH85 and BLG94 lists; temperatures by SH85 were systematically corrected upward according to Table 2 of BLG94. The correction applied was on average $\sim+52 \mathrm{~K}$; BLG94 values were adopted for the 8 stars in common. The resulting sample of population I main sequence stars includes 57 objects and is shown in Table 7 . $B-V$, $(V-R)_{C},(V-I)_{C}$, and $(V-K)_{J}$ dereddened colors for these stars were collected from F89, SH85, BLG94 and Cousins (1980) data sets; they are shown in Columns 7, 8, 9 and 10 of Table 7, respectively. Separate semi-empirical calibrations were obtained for each color by fitting a second order polynomial to the $(V-R)_{C},(V-I)_{C}$, and $(V-K)_{J}$

$-T_{\text {eff }}$ data, and a third order polynomial to the $(B-V)-T_{\text {eff }}$ data, in the temperature range $5000<T_{\text {eff }}<8300 \mathrm{~K}$. The polynomial fits are given below and shown in Figure 3 (solid 
lines).

$$
\begin{gathered}
T_{\text {eff }}=-5718.4077(B-V)^{3}+10088.399(B-V)^{2}-9316.128(B-V)+9115.8314 \quad(4 a) \quad(57 \text { objects }) \\
T_{\text {eff }}=3350.38062(V-R)_{C}^{2}-9445.28906(V-R)_{C}+8757.94727 \quad(4 b) \quad(22 \text { objects }) \\
T_{\text {eff }}=753.906677(V-I)_{C}^{2}-4836.16016(V-I)_{C}+8801.4248 \quad(4 c) \quad(22 \text { objects }) \\
T_{\text {eff }}=300.313507(V-K)^{2}-2447.8403(V-K)+8768.1709 \quad(4 d) \quad(54 \text { objects })
\end{gathered}
$$

Deviations from the polynomial fittings are $\leq \pm 0.02$ mag in $V-R$ and $V-I$, and $\leq \pm$ $0.05 \mathrm{mag}$ in $B-V$ and $V-K$ (with $80 \%$ of the objects within $\pm 0.03 \mathrm{mag}$ ).

We have compared the coefficients of our polynomial fitting for the $(V-K)$ $T_{\text {eff }}$ calibration with BLG94 (lower line of BLG94 Table 8). In the temperature range $5700<T_{\text {eff }}<6700 \mathrm{~K}$ the two calibrations agree within $10 \mathrm{~K}$. The discrepancy is of about $40 \mathrm{~K}$ at $T_{\text {eff }}=5100$ and $7700 \mathrm{~K}$, and of about $100 \mathrm{~K}$ at $T_{\text {eff }} \sim 9000 \mathrm{~K}$, with our temperatures systematically cooler. Our calibration is extrapolated beyond $8300 \mathrm{~K}$, and hence not reliable. We estimate that equations (4) can be used to derive reliable $T_{\text {eff }}$ from observed $B-V$, $(V-R)_{C},(V-I)_{C}$, and $(V-K)_{J}$ colors within the temperature interval $5000<T_{\text {eff }}<8000 \mathrm{~K}$. The dashed lines in Figure 3 represent K92 theoretical color-temperature calibrations for the same metal abundance and gravity of the dwarf sample in Table 7 (i.e. $[\mathrm{A} / \mathrm{H}]=0.0$ and $\log g=4.0)$. We find that while theoretical and semi-empirical $(V-K)-T_{\text {eff }}$ calibrations are almost indistinguishable (upper panel of Figure 3), in the temperature range of our interest (i.e. around $6200 \mathrm{~K}$ ), the $B-V, V-R$ and $V-I$ theoretical calibrations lie systematically below their semi-empirical counterparts.

\section{The color- $T_{\text {eff }}$ transformations for RR Lyraestars}

We have corrected K92 synthetic colors to tie them to the semi-empirical calibrations on the assumption that the offset between synthetic and observed color is independent of gravity and metallicity 0 . We have calculated the offsets in color $\Delta(B-V), \Delta(V-R)$, and

\footnotetext{
${ }^{6} \mathrm{~A}$ comparison between the temperature calibrations of the $b-y$ index using K92 and the new OSMARCS
} 
$\Delta(V-I)$ between $\mathrm{K} 92$ synthetic colors at $[\mathrm{A} / \mathrm{H}]=0.0$ and $\log g=4.0$, and $\mathrm{K} 92$ colors for the gravity and the metallicity required for each star, and we have subtracted these offsets from the semi-empirical calibrations. We have thus generated a new set of K92 synthetic color-temperature transformations tied to the semi-empirical calibrations. From them we have read the temperatures corresponding to our spectra.

In Table 8 we list the temperatures of the average spectra derived from the various color indices. Temperatures for individual stars generally agree with each other within $200 \mathrm{~K}$ with $V-K$ and $V-R$ usually giving the cooler values. The scatter is much higher $(\geq 300 \mathrm{~K})$ if we use $T_{\text {eff }}$ from K92 models not corrected for the semi-empirical calibrations. The effective temperature derived from the $(B-V)$ color of VX Her is very discrepant and was not considered; VX Her and RR Lyr are the stars which have the oldest and least accurate photometry among our objects. Further, we did not use $T_{\text {eff }}$ from $B-V$ for $\mathrm{X}$ Ari since the $B-V$ color for this star is affected by the emission associated with the bump in the light curve (Gillet \& Crowe 1988). Incipient $\mathrm{H}_{\alpha}$ emission was clearly detected in our spectra. V445 Oph exhibits a large discrepancy between temperatures derived from different colors. This is the most reddened object in our sample and some uncertainty exists on the reddening and on the appropriate absorption coefficient $\mathrm{R}_{V}$ to use for this star. The metallicity of the star is controversial too (see the discussion in Cacciari et al 1992). We have iteratively tried various combinations of reddening, $\mathrm{R}_{V}$ and metallicity and used in the end the combination $E(B-V)=0.27, \mathrm{R}_{V}=3.1$ and $[\mathrm{Fe} / \mathrm{H}]=+0.2$, which seems to give more consistent temperatures if we do not consider the $T_{\text {eff }}$ derived from $V-K$. On the other hand, if we compare the dereddened colors of V445 Oph and SW And (which according to their $\Delta \mathrm{S}$ should have similar metallicities), we see that while $B-V, V-R$ and $V-I$ colors of the two stars in the phase range of our spectra (i.e. $0.5<\phi<0.7$ ) agree within $\pm 0.02 \mathrm{mag}$, the $V-K$ color of V445 Oph is 0.1 mag redder than $\mathrm{SW}$ And one. On the basis of these considerations we chose not to use the $V-K$ temperature of V445 Oph. The temperatures not used are given in parenthesis in Table 8.

models (Edvardsson et al 1993) actually suggests a quite different metallicity dependence, likely due to a different treatment of convection between these two latest generation model atmospheres. Unfortunately, we do not have predictions of $V-K$ colors with the OSMARCS models. However, the $V-K$ colors may be empirically calibrated using the temperatures by Edvardsson et al (1993) and Nissen et al (1994; these last authors use the same procedure); temperatures obtained by this procedure are similar to those obtained by the K92 calibration for population I stars, but they are much lower (up to $250 \mathrm{~K}$ ) for the most metal-poor stars, yielding much lower $[\mathrm{Fe} / \mathrm{H}]$ values (by up to $0.4 \mathrm{dex}$ ). This would exacerbate the discrepancy between abundances for globular clusters derived from giants and RR Lyraes (see Section 5.1.1). We then decided to consistently use K92 models (which are the only latest generation model atmospheres available for RR Lyrae) for the color- $T_{\text {eff }}$ calibrations throughout this paper. 
Since the $V-K$ - temperature calibration is not affected by any discrepancy between synthetic and observed colors and it is less metallicity dependent (see F89 and references therein), we adjusted the temperatures derived from $B-V, V-R$ and $V-I$ to the $V-K$ one by correcting them for the systematic mean differences between temperatures derived from individual colors and $V-K(-49 \mathrm{~K},-51 \mathrm{~K}$ and $-97 \mathrm{~K}$ for the $B-V, V-R$ and $V-I$ colors, respectively). Finally, average temperatures were calculated as the mean of these corrected $T_{\mathrm{eff}} \mathrm{s}$; they are listed in Column 6 of Table 8 together with the related r.m.s. (Column 7). The typical internal uncertainty is $25 \mathrm{~K}$.

Besides the internal errors, uncertainties in the derived temperatures depend on: (i) the assumed gravities and metal abundances; (ii) uncertainties in the assumed reddening; (iii) the accuracy of the photometric data; and (iv) the theoretical soundness of using static model atmospheres to reproduce the dynamical atmospheres of pulsating variables (this last issue is discussed in the next subsection). Uncertainties in the assumed gravity affect the color-temperature transformations only marginally; in fact, irrespective of metallicity and color index, a variation in $\log g$ from 2.5 to 3.0 produces a variation in the temperatures derived from K92 models generally $\leq 40 \mathrm{~K}$ (see also Figure 3 of Liu \& Janes 1990). To enter K92 models we have assumed, therefore, a constant gravity $\log g=2.75$ as appropriate for all stars; this value corresponds to the average of Column 3 in Table 10. The sensitivity of the color-temperature transformations to the input metal abundance has been checked for a metal-rich and a metal-poor object. Tests made on SW And $([\mathrm{Fe} / \mathrm{H}]=-0.10)$ and VY Ser $([\mathrm{Fe} / \mathrm{H}]=-1.75)$ show that the effect is relevant only for temperatures derived from $B-V$ and $V-R$, and only for metal-rich objects. In fact, a variation of 0.3 dex in metallicity translates into a variation of the estimated temperature from $B-V$ of $\sim 100 \mathrm{~K}$, and from $V-R$ of $\sim 50 \mathrm{~K}$, at $[\mathrm{Fe} / \mathrm{H}]=-0.10$, or $\sim 30 \mathrm{~K}$ and $\sim 6 \mathrm{~K}$ respectively at $[\mathrm{Fe} / \mathrm{H}]=-1.75$. For the other colors the effect is $\leq 10 \mathrm{~K}$. Recall that the reddening accuracy is $\pm 0.02 \mathrm{mag}$ in $E(B-V)$ (i.e. $\sim 100 \mathrm{~K})$; we can safely assume that the errors due to the colors are $\leq 0.01$ mag (i.e. $\sim 50 \mathrm{~K}$ ), since we read colors from smoothed curves where the accuracy of single data points is $\pm 0.01 \mathrm{mag}$. These contributions add in quadrature and lead to an error on the derived $T_{\text {eff }}$ of $\sim 115 \mathrm{~K}$. The adopted temperatures are listed in Column 2 of Table 10 .

As we have discussed in Section 2.4, RR Lyr and ST Boo exhibit the highest discrepancy between phases estimated from ephemerides and phases derived from radial velocities measured from the average spectra. We find that if these last values are used, the adopted gravities should be lowered by 0.10-0.15 dex for both stars and the adopted temperatures should be $\sim 40 \mathrm{~K}$ and $60 \mathrm{~K}$ cooler for ST Boo and RR Lyr respectively; these values are within the uncertainties adopted for these atmospheric parameters.

\section{$T_{\text {eff }}$ 's from $\mathbf{H}_{\alpha}$ profiles}


The procedure we have followed to derive effective temperatures from the observed colors of the program stars, and the abundance analysis technique we will describe in Section 4, are based on the assumption that K92 model atmospheres are able to reproduce well the RR Lyrae variables at minimum light. In the following we shall briefly discuss this assumption. Figure 3 shows that some discrepancy exists between the observed and K92 synthetic colors for dwarf stars. This problem is enhanced for RR Lyraestars where differences between temperatures derived from color calibrations obtained by K92 models not corrected for the empirical calibration may rise to more than $300 \mathrm{~K}$. The discrepancy is reduced to less than $80 \mathrm{~K}$ (although not totally eliminated) by correcting for the semi-empirical calibrations derived for dwarf stars.

An independent estimate of the temperature of the continuum forming region is provided by the profile of the $\mathrm{H}_{\alpha}$ absorption line. Our Palomar spectra are not well suited to this purpose, owing to the quite large uncertainties in the location of the continuum level in this spectral region due to the presence of strong diffraction fringes, and to the narrow free spectral range. We therefore acquired additional spectra (resolution $\sim 15,000$ and $S / N \sim 130$ ) for three of the program stars (SW And, RR Cet, and X Ari) using the REOSC Echelle spectrograph at the $182 \mathrm{~cm}$ Copernicus reflector of Asiago Astrophysical Observatory. This spectrograph uses a $79 \mathrm{gr} / \mathrm{mm}$ echelle grating (so that the free spectral range is 1.6 times that at Palomar) and a large format front illuminated Thomson CCD detector, with no appreciable diffraction fringes. Spectra of several subdwarfs were also acquired during the same runs with the same instrumental set-up, but higher $S / N(>250)$ : some of them were used here to further compare our $T_{\text {eff }}$ from $\mathrm{H}_{\alpha}$ profiles with those derived from our calibration of photometric indices, as well as those obtained by Fuhrmann et al. (1994) also from $\mathrm{H}_{\alpha}$ profiles.

All Asiago spectra were reduced using a homogenous procedure. We find that after flat fielding by means of the spectra of a quartz iodine lamp reflected by the dome, a residual trend of the continuum with wavelength still existed. This trend repeated very regularly through different orders, so that it was efficiently eliminated by using the same average pseudocontinuum (obtained by automatic IRAF routines) for all orders. We attribute this effect to the different way the slit is illuminated when using light from the star or from the lamp. The spectra were then wavelength calibrated using a Th lamp, and telluric lines were divided out by a synthetic spectrum constructed using wavelengths and equivalent widths from Moore et al. (1966)凹.

$\mathrm{H}_{\alpha}$ profiles obtained by this procedure were then compared with synthetic profiles

\footnotetext{
${ }^{7}$ Line intensities were adjusted to fit telluric lines detected in each individual spectra.
} 
computed using K92 models and our own spectral synthesis code, which included Doppler, natural damping, resonance (Ali \& Griem 1965, 1966), and Stark (Vidal et al 1970) broadening, following prescriptions similar to those adopted by Fuhrmann et al. (1993, 1994). These prescriptions should be correct for electron densities above $10^{12}$ electrons per $\mathrm{cm}^{3}$ (roughly corresponding to $\log \tau \sim-2$ for the RR Lyrae variables). However, line formation in the outer part of the atmospheres is likely affected by appreciable deviations from LTE, as demonstrated by the presence of some incipient emission at wavelengths slightly shorter than the core of the lines: hence these part of the profiles (as well as those contaminated by other lines) were not considered in the $T_{\text {eff }}$ derivations. A comparison with the solar flux spectrum (Kurucz et al 1984) showed that our computed profiles are a little bit too narrow, so that the solar $T_{\text {eff }}$ we derived is $6017 \mathrm{~K}$. We then systematically corrected downward our $T_{\text {eff }}$ 's by $247 \mathrm{~K}$. Following this procedure, our $T_{\text {eff }}$ 's are relative to the Sun. We note that the profiles used for the RR Lyrae variables were computed assuming $\log g=2.75$ and $[\mathrm{A} / \mathrm{H}]=-1$; however, this assumption is not critical since Fuhrmann et al (1993) have shown that when opacity is dominated by $\mathrm{H}^{-}$and most electrons are provided by $\mathrm{H}$, the $\mathrm{H}_{\alpha}$ profile is almost independent of surface gravity and metal abundance.

A comparison of observed and computed profiles is given in Figure 4. Our $T_{\text {eff }}$ 's from $\mathrm{H}_{\alpha}$ profiles are listed in Table 9 both for the three RR Lyrae variables and the seven subdwarfs. In this table we also give $T_{\text {eff }}$ 's from our photometric calibrations and those deduced by Fuhrmann et al (1994) from $\mathrm{H}_{\alpha}$ profiles. On average, our $\mathrm{H}_{\alpha} T_{\text {eff }}$ 's for the dwarfs are slightly larger than both those derived from colours and by Fuhrmann et al; however differences $(27 \pm 41 \mathrm{~K}$ and $32 \pm 34)$ are not significant. The r.m.s. scatter of the star-to-star residuals (108 and $89 \mathrm{~K}$ respectively) indicates that the internal errors are $\sim 100 \mathrm{~K}$; we attribute these errors to small $(\sim 1 \%)$ uncertainties in the location of the continuum level. In the case of the RR Lyrae variables, the average difference between $T_{\text {eff }}$ 's from $\mathrm{H}_{\alpha}$ profiles and those derived from the photometric indices is $-73 \pm 75 \mathrm{~K}$ : again this difference is not significant. The scatter of the results for individual stars $(132 \mathrm{~K})$ is consistent with an error bar similar to that obtained for dwarfs.

We conclude that the comparison with the $H_{\alpha}$ profiles supports our color-temperature calibrations, and confirms that K92 models are able to reproduce the temperature stratification of the continuum forming region $(\log \tau>-2)$ of an RR Lyrae at minimum light to within an accuracy of $\sim 150 \mathrm{~K}$.

\section{K92 models and the atmospheres of RR Lyrae stars at minimum light}

The suitability of K92 models to reproduce the regions of formation of the weak metal lines used in the abundance analysis $(\log \tau \sim-1 \div-2)$ for RR Lyrae stars close to the minimum 
light is demonstrated by their capability to well reproduce the ionization and excitation equilibrium conditions for elements of the iron group as well as for the $\alpha$-elements (see Section 4), with no need for deviations from LTE conditions. Deviations from LTE, possibly induced by shock waves propagating throughout the atmosphere of the RR Lyraes are perhaps required only to explain the anomalous behaviour of some peculiar elements (see Section 4.4); however, only small amplitude shock waves are required, which are not expected to alter the global atmospheric structure of the star. Beyond these regions (i.e. at $\log \tau<-2$ ) we do not have good tests available to check whether K92 model atmospheres are still appropriate; however, the asymmetry exhibited by the core of the $\mathrm{H}_{\alpha}$ line, as well as its large variations from one spectrum to the other, would suggest that deviations from LTE take place in the outer atmospheric regions.

To conclude, as far as abundance or B-W analyses are concerned, i.e. as far as the regions of interest are confined to $\log \tau=0 \div-2$, K92 models seem adequate to reproduce the atmospheres of RR Lyraestars at minimum light. They certainly represent an improvement upon K79 model atmospheres which gave excitation and ionization temperatures higher by about $250 \mathrm{~K}$ than temperatures derived from colors. The comparison between K92 and K79 models is shown in Figure 5.

\subsection{Microturbulent velocities}

Microturbulent velocities $v_{\mathrm{t}}$ were determined by zeroing the slope of abundances with $E W \mathrm{~s}$ for individual Fe I lines. Since $E W$ s were deduced for numerous lines on both the linear (which is very extended in the spectrum of RR Lyrae variables) and flat parts of the curve of growth, the values of $v_{\mathrm{t}}$ can be accurately determined from our spectra: typical internal errors are $\pm 0.2 \mathrm{~km} \mathrm{~s}^{-1}$.

An assumption inherent to these determinations of $v_{\mathrm{t}}$ is that the microturbulent velocity is constant throughout the stellar atmosphere. There is no sound argument supporting this assumption apart simplicity; in fact, non-constant microturbulent velocities have been proposed for the Sun (see e.g. Maltby et al. 1986), and the presence of shock waves makes it likely that $v_{\mathrm{t}}$ is not constant in the atmospheres of the RR Lyrae stars too. In the following we explore more in detail the aftermath of the assumption of constant $v_{\mathrm{t}}$.

The run of $v_{\mathrm{t}}$ with optical depth is difficult to be determined since it cannot be presently derived on a theoretical base alone. On the other side, very high resolution, high $\mathrm{S} / \mathrm{N}$ data are required to disentangle the contributions of micro and macro-turbulence from 
line profiles. The only data available for RR Lyrae variables are the $E W \mathrm{~s}$; we then tried to estimate the range of possible variations of $v_{\mathrm{t}}$ within the atmosphere of a typical RR Lyrae star at minimum light from the trends of the abundances deduced from individual lines with $E W \mathrm{~s}$, exploiting the fact that the depth of formation of lines is a function of line strength. In fact, if $v_{\mathrm{t}}$ is not constant throughout the atmosphere, we expect that significant trends may be present in the run of abundances with line strength even though the average slope has been set at zero by adopting a suitable constant value for $v_{t}$.

In Figure 6a we plotted the residuals of the abundances deduced from individual Fe lines minus the average Fe abundances, against $E W \mathrm{~s}$ (in order to improve statistics, we considered all 10 program stars together). The scatter of individual points in Figure 6a is rather large; however, the correlation of these residuals with a quadratic least square relation is highly significant (about 5 standard deviations), while as expected the linear correlation coefficient is very close to zero; this means that significant trends are indeed present in the run of abundances with $E W \mathrm{~s}$, even though the average slope is zero. It should be noticed that deviations from zero are not large; this is shown by Figure 6b, where we plotted the average values of residuals computed in bins of 0.2 dex, against $E W \mathrm{~s}$ : the largest deviations are $<0.1$ dex. Similar small trends could well be due to causes other than systematic variations of $v_{\mathrm{t}}$ with optical depth, like e.g. deviation of observed line profiles from gaussians (used to derive the $E W \mathrm{~s}$ ), inappropriate consideration of damping, numerical approximations in the analysis code, etc. Hence, we do not think that the plots of Figures $6 \mathrm{a}$ and $6 \mathrm{~b}$ conclusively show that $v_{\mathrm{t}}$ changes with optical depth in the atmospheres of RR Lyrae variables at minimum light.

Anyway, in order to estimate the impact of these possible changes on our abundance derivations we must have at least an order of magnitude estimate of their values. This was obtained by comparing the run of Figure $6 \mathrm{~b}$ with those given by a standard abundance analysis (with constant microturbulent velocity) performed on $E W \mathrm{~s}$ of synthetic lines forming in a model atmosphere having $T_{\text {eff }}=6200 \mathrm{~K}, \log g=2.75$, and $[\mathrm{A} / \mathrm{H}]=-1.3$ (these values being assumed as representative of an RR Lyrae star at minimum light). Various $v_{\mathrm{t}}(\tau)$ laws, where $v_{\mathrm{t}}$ varies quadratically with log optical depth, were considered when computing synthetic profiles (quadratic laws were considered because they resemble that adopted by Maltby et al 1986 for the Sun). We finally selected for our comparison those cases in which the analysis of the synthetic lines yields a (constant) value of $v_{\mathrm{t}}=4 \mathrm{~km} \mathrm{~s}^{-1}$ and an amplitude of the run of the abundance residuals with $E W$ s similar to that obtained in our analysis of RR Lyrae stars at minimum. These runs obtained from synthetic lines are also shown in Figure 6b. Qualitatively, we found that a suitable quadratic $v_{\mathrm{t}}(\tau)$ law matches reasonably well the observations. The correct amplitude and the position of the minimum at $\log E W / \lambda \sim-4.6 \div-4.7$ are reproduced by adopting a minimum value of 
$v_{\mathrm{t}} \sim 0.6 \mathrm{~km} \mathrm{~s}^{-1}$ at a very shallow optical depth $(\log \tau \sim-3)$, and a large variation of $v_{\mathrm{t}}$ with optical depth $\left(v_{\mathrm{t}} \sim 8 \mathrm{~km} \mathrm{~s}^{-1}\right)$ at $\log \tau \sim 0$. In fact, if we assume that the minimum value of $v_{\mathrm{t}}$ is deeper in the atmosphere, the observed amplitude of abundance variations with $E W \mathrm{~s}$ is reproduced by much smaller variations of $v_{\mathrm{t}}$ with optical depth, but the minimum in the run of abundances with $E W$ s shifts at $\log E W / \lambda>-4.5$, in disagreement with data. A value of $\log \tau \sim-3$ for the location of the minimum of $v_{\mathrm{t}}$ is similar to that assumed by Maltby et al., close to the temperature minimum in the solar atmosphere. On the other hand, the value of $v_{\mathrm{t}}$ at minimum (which is required in order to place the minimum in the run of abundances with $E W \mathrm{~s}$ at $\log E W / \lambda \sim-4.6 \div-4.7)$ seems quite low. This casts some doubts over the whole procedure.

Even though not entirely convincing, we now have a guess for the $v_{\mathrm{t}}(\tau)$ law. We then run our abundance analysis for a test star assuming this law of variation of $v_{\mathrm{t}}$, and then compared these results with those obtained assuming a constant $v_{\mathrm{t}}$. The differences between the abundances obtained in the two cases yield a rough estimate of the possible influence of the assumption of a constant $v_{\mathrm{t}}$ on our final abundances. These differences are given in Column 7 of Table 11; in most cases they are less than 0.1 dex. Elements with few, very strong lines exhibit larger variations $(\sim 0.15 \div 0.20 \mathrm{dex})$, which are very sensitive to line strength (e.g. variations have the opposite sign for Ba II lines).

We conclude that present data do not warrant the adoption of a variable $v_{\mathrm{t}}$ with optical depth in our analysis; however, would $v_{\mathrm{t}}$ be indeed variable, we do not expect variations in the deduced abundances larger than 0.1 dex, except for those elements for which we measured $E W$ s for only a few, strong lines; in these cases, variations as large as $0.15 \div 0.20$ dex may be expected.

\subsection{Adopted atmospheric parameters}

The adopted atmospheric parameters are summarized in Table 10. Gravities, $T_{\text {eff }}$ and microturbulent velocities are as discussed in Sections 3.2, 3.4 and 3.5. Metallicities are instead slightly different than the values discussed in Section 3.3. They were obtained iteratively during the abundance analysis procedure and are close to the final derived values of $[\mathrm{Fe} / \mathrm{H}]$.

\section{ABUNDANCE ANALYSIS}


Abundances were derived from the $E W$ s using model atmospheres extracted from the grid by K92. Table 11 shows the sensitivity of the derived abundances on the values adopted for the atmospheric parameters. Values in Table 11 were obtained by comparing the derived abundances for RR Cet with those obtained by varying the atmospheric parameters one at a time within the estimated range of confidence. The main effects are those due to $T_{\text {eff }}$ on the abundances drawn from neutral species, and to $\log g$ on the abundances derived from singly ionized (dominating) species, while the overall metal abundance and the microturbulent velocity (which is typically very large in an RR Lyrae variable compared with a non-variable giant) play only a minor role. Sensitivity of element-to-element abundance ratios on the adopted atmospheric parameters are then much reduced (to $\leq 0.05 \mathrm{dex}$ ) if neutral species are compared with other neutral species (e.g. with Fe I), and singly ionized species with other singly ionized species (e.g. with Fe II): this is the adopted procedure throughout this paper. However, there are significant exceptions: the abundances derived from high excitation lines of dominant species (O I, Si II, S I) are sensitive to both gravity and temperature (with a trend opposite to that of Fe I lines); the abundance derived from a single, strong (resonance) line like that of Al I is very sensitive to the microturbulent velocity. Abundance ratios to Fe derived from lines of these species have then larger uncertainties $(\sim 0.10-0.15 \mathrm{dex})$.

Column 6 of Table 11 shows the variation of the solar reference abundances (obtained using the same line list used for the program stars) when the constant flux solar model atmosphere by K92 is replaced by Holweger \& Müller (1974; hereinafter HM) empirical model atmosphere, which is considered to be the best representation of the solar photosphere. Although the values in this column depend somewhat on the adopted line list, they provide a rough estimate of how the uncertainties in the abundances are related to the structure of the atmospheres of metal-rich non-variable stars. These values are generally small $(<0.06$ dex), and testify the significant progress made with the new generation of atmospheric models. For consistency, hereinafter we will use the abundances obtained from Kurucz models.

\subsection{Fe abundances}

Table 12 lists Fe abundances derived for the program stars. As mentioned in the Introduction, the determination of abundances for RR Lyraestars offers a unique chance to test empirically the reliability of abundances determined from high dispersion analysis; furthermore, departures from LTE might be large in the warm and rarified atmospheres 
of RR Lyrae variables. We expect that inadequacies of the assumptions (LTE, structure of the model atmospheres, etc.) will show up as trends of the derived abundances from individual lines with excitation and ionization, as well as element-to-element abundance ratios not fitting in the usual pattern observed in other old stars. However, in order to derive significant estimates of these inconsistencies, considerable care must be devoted to the derivation of an accurate set of oscillator strengths $g f_{\mathrm{s}}$; some insight can be obtained by a comparison with solar abundances. For this reason, results obtained for the Sun with the same code and set of $g f$ s are also given in Table 12.

\subsubsection{The Solar Fe abundance}

The solar Fe abundances listed in Table 12 require some more comments; in fact, in the last years there has been some debate about the determination of the solar photospheric Fe abundance, since the value obtained using precise $g f_{\mathrm{s}}$ from the absorption experiments of the Oxford group combined with laboratory determinations of the damping parameters and the HM model atmosphere $(\log \epsilon(\mathrm{Fe})=7.63$ : Simmons \& Blackwell 1982) is larger than those obtained from Fe II lines $(\log \epsilon(\mathrm{Fe})=7.48$ : Holweger et al 1990; $\log \epsilon(\mathrm{Fe})=7.54$ : Biémont et al 1991; $\log \epsilon(\mathrm{Fe})=7.48$ : Hannaford et al 1992) and from meteorites $(\log \epsilon(\mathrm{Fe})=7.51$ : Anders \& Grevesse 1990). New sets of $g f$ s for Fe I lines, based on accurate lifetimes and branching ratio measurements, have been published by Bard et al (1991), O'Brian et al (1991), and more recently by Bard \& Kock (1994). These sets of $g f_{\mathrm{s}}$ agree very well with each other: mean difference over the 29 strongest lines is $0.006 \pm 0.008$ dex, O'Brian et al $g f$ s being slightly smaller than those by Bard and coworkers, with a standard deviation of 0.044 dex for individual lines. The scatter is larger $(\sim 0.15$ dex $)$ for weaker lines, likely due to random errors in the branching ratios used by O'Brian et al (since a solar analysis using this set of $g f_{\mathrm{s}}$ for weak lines gives a large scatter), but the overall agreement is still good. The solar Fe abundance determined using $g f_{\mathrm{S}}$ from Bard et al (1991) is much closer to the meteoritic value $(\log \epsilon(\mathrm{Fe})=7.47$ : Holweger et al 1991) than that derived by Simmons \& Blackwell; however, lines used in that analysis are strong, most of them are not clean from blends, and the derived abundances depend on assumptions about damping, which are uncertain, and for which an average value was derived from the solar analysis itself. To have a better insight into this aspect, we redetermined the photospheric Fe abundances using the $E W$ s and $g f_{\mathrm{s}}$ by Simmons \& Blackwell (1982) and Holweger et al (1991), as well as lines with $g f_{\mathrm{s}}$ measured by Bard \& Kock (1994) and $E W$ s from the list of clean lines in the solar intensity spectrum by Rutten \& Van den Zalm (1984). This last group consists of lines which are much weaker than those used by Holweger et al (1991) and Simmons 
\& Blackwell, and hence less dependent on the assumption about collisional damping (for which we used a mean relation drawn from the Simmons \& Blackwell data when reanalysing all three sets of Fe line data). The mean Fe solar abundances provided by these three groups of lines (i.e., Simmons \& Blackwell 1982; Holweger et al 1991; and Bard \& Kock 1994 with Rutten \& Van den Zalm 1984) using our code and the K92 solar atmosphere with a depth-independent microturbulent velocity of $0.9 \mathrm{~km} \mathrm{~s}^{-1}$ were $\log \epsilon(\mathrm{Fe})=7.59,7.50$, and 7.52 respectively. We consider the last of our redeterminations $(\log \epsilon(\mathrm{Fe})=7.52$; which agrees very well with the meteoritic value) to be the most reliable. Therefore the following procedure was adopted in this paper: whenever possible, $g f \mathrm{~s}$ for the Fe I lines detected in the spectra of our targets were taken from papers of the Oxford group (see, for references, Simmons \& Blackwell, 1982) and Bard et al (1991), [we could not use Bard \& Kock (1994) because lines in their list are too weak to be measured in the spectra of the RR Lyraes]; the $g f$ s from the Oxford group were corrected upward by 0.03 dex to account for the systematic difference with those by Bard et al. This systematic difference might be attributed to a small error in the zero-point of Oxford $g f s$, which anyhow must be ultimately based on lifetime measurements. $g f \mathrm{~s}$ for Fe II lines were taken from Heise \& Kock (1990), Biémont et al (1991) and Hannaford et al (1992). For all remaining lines (taken from the list by Rutten \& van der Zalm 1984, and Blackwell et al 1980), solar $g f_{\text {s }}$ were derived using the HM model atmosphere and the Fe abundance derived from the other lines. As shown in Table 12 the solar Fe abundance obtained with this set of $g f$ s for Fe I is slightly larger than that derived from Fe II lines; however, this difference is small when using the K92 model and does not cause serious concern; the trend with excitation potential is also very small in the Sun.

\subsubsection{Trends with excitation and ionization}

On the whole, the observed trends for Fe abundances measured in RR Lyrae stars are very small, and not significant; they are well within the uncertainties in the atmospheric parameters. The average difference between Fe abundances obtained from neutral and singly ionized lines is $0.05 \pm 0.03 \mathrm{dex}(\sigma=0.10 \mathrm{dex})$, in the sense that abundances given by neutral lines are smaller, that is, this difference has the opposite sign of that obtained for the Sun. However, if we remove from this comparison ST Boo and VX Her, for which no $\mathrm{B}-\mathrm{W}$ analysis is available, the mean difference drops to $0.01 \pm 0.02 \operatorname{dex}(\sigma=0.06 \operatorname{dex}$, 8 stars). If differential abundances with respect to the Sun are considered, $[\mathrm{Fe} / \mathrm{H}]$ values

derived from neutral lines are on average lower than those derived from singly ionized lines by $0.11 \pm 0.03$ dex. The mean slope in the abundance vs excitation potential is 
$0.000 \pm 0.008 \mathrm{dex} / \mathrm{eV}(\sigma=0.024 \mathrm{dex} / \mathrm{eV})$, and it is consistent with the value obtained for the Sun. The trend with ionization could be eliminated by raising all $T_{\text {eff }}$ for the program stars by $70 \mathrm{~K}$, which is well within the uncertainties of the adopted temperature scale. The observed scatter is compatible with internal errors of $\pm 80 \mathrm{~K}$ in $T_{\text {eff }}$ (due e.g. to errors of $0.015 \mathrm{mag}$ in $E(B-V))$ and to errors of \pm 0.15 dex in $\log g$, once the error in the determination of abundances from individual lines $(0.15 \mathrm{dex})$ is taken into account. Internal errors in the abundances deduced from neutral and singly ionized Fe lines are 0.08 and 0.09 dex respectively: then, it seems that the best estimate of $[\mathrm{Fe} / \mathrm{H}]$ (to be compared e.g. with photometric data) is obtained by averaging these two values.

Table 11 reports the effects on the abundance derived for each element of the variation of the atmospheric parameters within their quoted uncertainties, as well as the effects due to a variation of the solar reference abundances and to the use of a turbulence velocity varying with optical depth. This table thus provides an estimate of the systematic errors affecting our abundance analysis. These systematic errors add to 0.12 and 0.08 dex for FeI and FeII respectively, on average are of about 0.11 dex, and only in a few cases (O I and Al I) can be as large as 0.26 dex.

\subsubsection{Comparison with results from statistical equilibrium computations}

Combining possible systematic errors in the temperature and gravity scales and in the $g f_{\mathrm{s}}$, we get an upper limit of $\sim 0.2$ dex for the systematic difference between abundances given by neutral and singly ionized lines, due to some overionization; on the other hand, there is no evidence for departures from Boltzmann excitation equilibrium at $\sim 0.1-0.2$ dex level. These observational data can be compared with predictions from statistical equilibrium calculations. The aim here is to constrain the relevance of collisions in this kind of computation. Once properly calibrated, these cross sections can thus be used in computations appropriate for stars in other evolutionary phases (subdwarfs, metal-poor RGB stars). To this purpose we used the 61-level Fe I model atom by Gratton et al (1995). This model atom reproduces quite closely Steenbock's (1985). Given the uncertainty in the collisional cross sections, we adopted a parametric approach: we assumed that the overall transition rate due to collisions $c$ is represented by the sum of two terms due to collisions with electrons $\left(c_{e}\right)$ and with neutral hydrogen atoms $\left(c_{H}\right)$, respectively. The last one was obtained following the approach by Drawin $(1968,1969)$; we then may write (see Gratton et al 1995):

$$
c_{H}=k f\left(\frac{m_{A}}{m_{H}}, T, \frac{p_{g}}{p_{e}}\right) c_{e}
$$


where $m_{A}$ and $m_{H}$ are the atomic weights for the considered atom $(\mathrm{Fe})$ and that of hydrogen, $T$ is the temperature, $p_{g}$ and $p_{e}$ are the gas and electron pressures, and $k$ is a free parameter that we empirically calibrate in order to model observations for RR Lyraestars. This formalism is adopted in order to reflect the different degree of uncertainty present in the cross sections for collisions with electrons (sometimes rather well known, as in the case of $\mathrm{Na}$, though not of $\mathrm{Fe}$ ) and with neutral hydrogen atoms (always very poorly known). Note however that in several cases (including $\mathrm{Fe}$ ) $k$ is better interpreted as an estimate of the overall relevance of collisions; in fact, we may write:

$$
c=c_{e}\left[1+k f\left(\frac{m_{A}}{m_{H}}, T, \frac{p_{g}}{p_{e}}\right)\right] .
$$

Results of our statistical equilibrium computations made using MULTI code by Scharmer \& Carlsson (1985) are shown in Figures 7a,b, which compare departure coefficients obtained with $k=31.6$ and $k=0$ for a typical atmosphere of an RR Lyrae star close to minimum $\left(T_{\text {eff }}=6200 \mathrm{~K}, \log \mathrm{g}=2.75,[\mathrm{~A} / \mathrm{H}]=-1.3\right)$. A large Fe overionization is present deep in the atmosphere in the second case, while the overionization is significant only at shallow optical depths (above the region of formation of most lines) in the first case. We then synthesized line profiles using populations calculated by this statistical equilibrium (non-LTE) computations, and then reanalyzed the resulting equivalent widths by assuming LTE. Figure 8 shows the differences between abundances obtained from LTE analysis of neutral and singly ionized Fe lines as resulting from this exercise, for different values of $k$. The range allowed for these differences by observational errors and uncertainties in the adopted atmospheric parameters is shown by the shaded region in Figure 8. From this figure, we derive $k \geq 30$. However, given the large uncertainties in all collisional cross sections for $\mathrm{Fe}$, the correct interpretation of this result is that collisional cross sections are much larger than originally assumed in our Fe I model atom, rather than that collisions with neutral $\mathrm{H}$ atoms are much more important than collisions with electrons. Anyway, if the collisional cross sections determined by this procedure are used in the computation of statistical equilibrium for subdwarfs and metal-poor RGB stars, it turns out that corrections to LTE abundances for Fe are negligible for these stars, supporting results of classical LTE analyses. The results of detailed computations will be given in Gratton et al (1995).

\subsubsection{Comparison with previous results}

We have compared our abundances with those derived previously for the same stars. We have four stars in common with Butler (1975) and Butler \& Deming (1979). Our 
abundances for these variables are systematically lower by a constant offset; the differences are $0.18 \pm 0.04 \operatorname{dex}(\sigma=0.07 \mathrm{dex})$, and $0.17 \pm 0.07 \operatorname{dex}(\sigma=0.13 \mathrm{dex})$, with Butler and Butler \& Deming respectively. Butler \& Deming abundances were derived using only singly ionized lines, thus a more appropriate comparison is made with our abundances from Fe II lines. In this case the mean difference is $0.14 \pm 0.08 \operatorname{dex}(\sigma=0.16 \operatorname{dex})$. A direct comparison of the atmospheric parameters is not possible since Butler (1975) used a curve-of-growth method, while Butler \& Deming spectra were taken at a very different phase due to observational limitations. We have estimated temperatures at phases corresponding to Butler \& Deming spectra using our color-temperature calibrations. We found that these temperatures are about $500 \mathrm{~K}$ cooler than Butler \& Deming's for SW And and X Ari, while the $T_{\text {eff }}$ of RR Cet is $30 \mathrm{~K}$ cooler. The adoption of such cooler temperatures would result in Fe abundances $\sim 0.2$ dex lower than those obtained by Butler \& Deming, bringing them into agreement with the present paper Fe abundance estimates.

We may also compare our Fe abundances for VY Ser with those determined by Carney \& Jones. As mentioned in Section 2.3, EW and atmospheric parameters adopted by these authors are close to ours. The agreement is good: Carney \& Jones found $[\mathrm{Fe} / \mathrm{H}]=-1.82$ and -1.73 from neutral and singly ionized lines; our values are $[\mathrm{Fe} / \mathrm{H}]=-1.78$ and -1.64 .

\subsubsection{Abundances at maximum light}

For one of the program variables (SW And) we have acquired spectra at phases close to both minimum and maximum light. The latter were taken to study the H-emission which is often associated with the hump in the light curve of the ab-type RR Lyraes, a feature that roughly corresponds to $\phi \sim 0.9$ of the pulsation.

To address a referee's comment we analyzed and compared the abundances derived from the two sets of spectra. However, it should be mentioned that serious concern arises when the region close to maximum light is used for abundance analysis of RR Lyraestars. This is, in fact, a region of fast acceleration where magnitude, colors, radial velocity and hence temperature and gravity of the variable, change by a large amount in a very short time. Shock waves propagate through the continuum and line forming regions during this part of the pulsation cycle, one of which, the so called main-shock (Fokin 1992), is associated with the hump in the light curve. The energy released by the main shock produces H-emission and UV-excess that can still be detected some time after the hump itself (Preston \& Paczynsky 1964, Gillet \& Crowe 1988, Clementini et al 1994b). Under such circumstances strong departure from LTE conditions and moreover important modifications 
of the atmospheric structure, are expected to take place, making the use of LTE model atmospheres in hydrostatic equilibrium probably no longer appropriate for high precision abundance analysis.

No H-emission has been detected, however, in our spectra of SW And close to maximum light. Indeed Preston \& Paczynsky (1964) found for SW And the weakest and shortest-lived $\mathrm{H} \gamma$-emission, among the five ab-type RR Lyraes they studied.

Effective parameters at phases close to maximum were derived following the same precepts adopted for spectra at minimum light. However, we must point out that in the phase range 0.85-1.00 $T_{\text {eff }}$ and log g changed by large amounts (up $\sim 500 \mathrm{~K}, \sim 0.5$ dex) while each single exposure was acquired, since typical exposures were of about 20-25 minutes, in order to get a reasonable $\mathrm{S} / \mathrm{N}$ ratio: therefore the adoption of a single value of $T_{\text {eff }}$ and $\log$ g during this part of the light-curve might be questioned. The adopted values are those corresponding to the phase of median energy within the range covered by each exposure. $T_{\text {eff }}$ 's at these phases are somewhat different from the values at mean exposure, depending on the part of the light-curve sampled; however, this choice reduced significantly the scatter in abundances deduced from individual spectra. The first part of Table 13 lists Fe abundances obtained from individual spectra corresponding to the region around maximum light : for comparisons, the values obtained at minimum light are also given. The average Fe abundances obtained from spectra at maximum $(\log \mathrm{n}(\mathrm{Fe})=7.50$ and 7.46 for Fe I and II respectively) are very close to the values obtained at minimum light (7.45 and 7.50); the r.m.s. scatters of individual values (0.10 and $0.05 \mathrm{dex})$ are quite small. However, inspection of Table 13 reveals that average Fe abundances from individual spectra are correlated with the slope of abundances deduced from Fe I lines with excitation potential. This suggests that the adopted $T_{\text {eff }}$ 's are not the best values: it should be noticed that for spectra taken during the raising part of the light-curve, rather large errors in $T_{\text {eff }}$ 's deduced from colors may be due to even small errors in the adopted phases, in view of the large time derivatives of colours. The second part of Table 13 gives the Fe abundances obtained when $T_{\text {eff }}$ 's deduced from colors are replaced by those obtained by forcing the slope of abundances deduced from Fe I lines with excitation potential to have the same value over the whole light curve. The average Fe abundances obtained with these $T_{\text {eff 's }}$ (7.47 and 7.46) are even closer to the values at minimum light than those obtained with $T_{\text {eff }}$ 's deduced from colors; furthermore, the scatter of values obtained from individual spectra is now reduced to 0.04 for both Fe I and Fe II, which is equal to the internal error determined from the line-to-line scatter in each spectrum. The small trend with phase in the difference between abundances from neutral and singly ionized lines might be attributed to small $(\sim 0.2$ dex $)$ errors in the adopted gravities: this errors are within the uncertainties of numerical integration of the radial velocity curve during the fast raise at maximum. 
The extension of this comparison to other elements shows that abundances deduced from spectra taken at maximum light usually agree very well with those obtained at minimum light: for most elements, differences are $<0.10$ dex if $T_{\text {eff }}$ 's are derived from the light curve, and $<0.08$ dex if they are deduced from the equilibrium of ionization for Fe I. Larger differences (in the sense abundances at minimum minus abundances at maximum light) were obtained only for $\mathrm{Na}(-0.24)$, $\mathrm{Ca}(-0.13)$, and $\mathrm{Mg}(+0.21)$ : these values were obtained using $T_{\text {eff }}$ 's from Fe I excitation, but they are very similar to those obtained using $T_{\text {eff }}$ 's from colors. Since these discrepancies are present only for neutral species, and correlates with ionization potential, we are inclined to attribute them to small deviations of the model atmospheres from real ones. Indeed, we think that the agreement between the abundances deduced from spectra obtained at the extremes of the light curve is astonishing, once the large variations of the atmospheres during these exposures are considered. On the whole, we think these comparisons strongly support the present abundance analysis.

\section{2. $\alpha$-elements}

Table 14 collects the results for the abundances of the $\alpha$-elements. The last column gives the average overabundances of all the elements listed in the table for each star, while the mean overabundances of $\alpha$-elements in metal-poor RR Lyrae stars $([\mathrm{Fe} / \mathrm{H}]<-1)$ are listed in Table 15. For comparison, the mean overabundances derived from accurate abundance analysis of other groups of metal-poor stars are also given in the last three columns of Table 15 :

(i) Column 5: 9 extremely metal-poor main sequence and subgiant stars recently measured by Nissen et al (1994)

(ii) Column 6: 20 metal-poor dwarfs and subgiants observed by Magain (1989) and Zhao \& Magain (1990)

(iii) Column 7: 13 metal-poor dwarfs and giants analyzed by Gratton \& Sneden (1991) and Carretta et al (1995).

Tables 14 and 15 show that all the $\alpha$-elements are overabundant in the observed metal-poor RR Lyrae variables, the mean overabundances being similar to those observed in non-variable less evolved stars (see also Figure 9). The star-to-star scatter of the average $\alpha$-element overabundances amongst metal-poor stars is small (0.06 dex) and is compatible with the hypothesis that all stars share the same enhancement of these elements. The scatter in element-to-element ratios is not large, and likely not significant.

Finally, it is worth mentioning the good agreement between the abundances obtained 
from neutral and singly ionized lines for $\mathrm{Si}$ : the mean difference is $-0.04 \pm 0.05$ dex ( $\sigma=0.12$ dex, 6 stars). This fact supports the LTE analysis. Conversely, our LTE analysis yields slightly larger $\mathrm{Ti}$ abundances from $\mathrm{Ti}$ II lines than those obtained from $\mathrm{Ti}$ I lines (the mean difference is $0.14 \pm 0.06 \mathrm{dex}, \sigma=0.18 \mathrm{dex}, 9$ stars), supporting a (small) $\mathrm{Ti}$ overionization. A similar suggestion was hinted by the analysis of metal-poor RGB stars by Gratton \& Sneden (1991).

\subsection{Fe-group and heavy elements}

Tables 16 and 17 list abundances for the Fe-group and heavy elements $(Z>28)$ in individual stars, while average overabundances in metal-poor $([\mathrm{Fe} / \mathrm{H}]<-1)$ variables are given in Table 18. For Sc II, Mn I, and Cu I lines, the hyperfine structure was considered in detail (see Gratton 1989, Gratton \& Sneden 1991, and Sneden et al 1991 for references). For comparison, abundances derived for metal-poor main-sequence and red giants (Gratton 1989, Gratton \& Sneden 1991, 1994, and Sneden et al 1991) are listed in Table 18. Main results are:

(i) Sc and Y abundances scale as Fe, in agreement with less evolved cooler stars. However, we obtained very low abundances for metal-rich variables (see next subsection).

(ii) Cr abundances derived from Cr II lines also scale as Fe. There is some indication of overionization of $\mathrm{Cr}$, since neutral lines give systematically lower abundances (by $0.25 \pm 0.06$ dex, $\sigma=0.20$ dex, 10 stars). A similar result was found by Gratton \& Sneden (1991) amongst the most metal-poor red giants.

(iii) Mn is found to be severely underabundant in metal-poor RR Lyraes; a less pronounced Mn underabundance was found in red giants (Gratton 1989). The larger effect seen in RR Lyraes may either be real (and in this case it would suggest a smaller contribution to nucleosynthesis by type I SNe), or may be the effect of some systematic deviation due to

atmospheric effects or departures from LTE in the formation of the Mn resonance lines (see also Gratton 1987).

(iv) The observed Ba abundances fit well in the pattern observed in other metal-poor stars.

\subsection{Sc and $Y$}

Abundances of some of the ionized species (Sc, Y, and rare earths) in metal-rich stars appear anomalous when compared to solar ones (which are typical of metal-rich objects). By 
combining data for SW And and V445 Oph, we get the results listed in Table 19. Inspection of this table shows that the abundances of Nd II, Ce II, Y II, and Sc II are anomalous P: no reasonable combination of errors in the atmospheric parameters may justify these results, which are confirmed by a comparison with synthetic spectra for some of the features of these species. For the remaining elements, there is no clear evidence for anomalies. Ca II is listed in Table 19 for its interest in the derivation of metallicities by means of the $\Delta \mathrm{S}$ and Ca II K line methods. Although we have not derived Ca abundances from singly ionized lines, the normal abundances obtained from neutral lines argue in favour of a normal strength for the Ca II lines. It is difficult to explain the underabundances of Nd II, Ce II, Y II, and Sc II by means of a nucleosynthetic argument; it is then interesting to explore the possibility that these anomalous abundances are due to an atmospheric effect that falsifies the results of our LTE analysis. We suspect that this mechanism may be related with the propagation of the shock waves connected with the pulsation, because non variable stars (but also Cepheids) with atmospheric parameters similar to RR Lyrae variables do not display Sc and Y underabundances (Luck \& Bond 1989; Luck et al 1990). $\delta$ Del stars (which are only slightly warmer than RR Lyraes at minimum: $T_{\text {eff }} \sim 7000 \mathrm{~K}$ vs $T_{\text {eff }} \sim 6300 \mathrm{~K}$ ) exhibit even overabundances of heavy elements. Unfortunately, accurate atomic data (cross sections etc.) for these elements are scarce, and do not allow to draw a very detailed model. We suggest that photoionization of these elements throughout most of the atmosphere might be due to photons emitted in the Lyman lines produced by the shock waves which propagate in the atmosphere of these variables (see Fokin 1992). We do not expect overionization of Ti II, Fe II, Si II, and Cr II, requiring Lyman continuum photons, which are abundant only in the very small regions immediately behind the shocks. Since Nd, Ce, Sc, and Y are more that $10^{9}$ less abundant that $\mathrm{H}$, this process is very minor from the point of view of the net energy balance: the total energy required to keep these elements twice ionized in the atmosphere of RR Lyraes is $\sim 310^{19} \mathrm{erg} / \mathrm{s}$ for a mass loss rate of $\sim 10^{-11} M_{\odot} / \mathrm{yr}$ (the energy required to ionize twice all $\mathrm{Sc}$ and $\mathrm{Y}$ in the atmosphere is $\sim 310^{25} \mathrm{erg}$ ). A priori, it is not clear why this mechanism is efficient for $\mathrm{Sc}, \mathrm{Y}, \mathrm{Ce}$, and $\mathrm{Nd}$, while it does not work for $\mathrm{Ca}$ and $\mathrm{Ba}$, why it is not balanced by recombinations, nor why it is not present in more metal-poor stars in our sample. Perhaps, this peculiar behaviour for $\mathrm{Ca}$ and $\mathrm{Ba}$ may be due to the small value of the photoionization cross section from the fundamental level of atoms and ions having an alkaline structure; these species have also a rather large jump to the first excited levels (3.13 eV for Ca II, $2.72 \mathrm{eV}$ for Ba II) ensuring that these last are scarcely populated. On the other hand, photoionization cross sections are much larger for atoms and ions having an alkaline-earth structure, like Sc II and Y II (which also have a

\footnotetext{
${ }^{8}$ Abundances from Nd II and Ce II lines were derived only for SW And.
} 
rather large number of low energy levels). Both hydrodynamical and statistical equilibrium computations are required to give a better insight into this issue.

\subsection{Mixing and abundances of light elements ( $\mathrm{C}, \mathrm{O}, \mathrm{Na}$ and $\mathrm{Al}$ )}

As mentioned in the Introduction, an anticorrelation between $\mathrm{Na}$ and $\mathrm{O}$ overabundances has been found for RGB stars in metal-poor globular clusters (see e.g. Sneden et al 1994 and references therein). Langer et al (1993) discussed various possible explanations for this anticorrelation, and reached the conclusion that the most favoured mechanism is the dredge-up of NO-cycle processed material related to meridional circulating currents, possibly activated by core rotation (Sweigart \& Mengel 1979). No such anti-correlation has been observed for RGB stars in metal-rich globular clusters (Brown \& Wallerstein 1992, Carretta \& Gratton 1992, Sneden et al 1994). This metallicity dependence is commonly attributed to the stronger molecular weight barrier existing in metal-rich giants (Tassoul \& Tassoul 1984), which inhibits the mixing. Observations of light elements (C, O, Na and $\mathrm{Al}$ ) in RR Lyraestars are of great interest because these stars are in an evolutionary phase following the RGB, and thus must have the envelope composition inherited from this previous evolutionary stage. Various features of $\mathrm{C}, \mathrm{O}, \mathrm{Na}$, and $\mathrm{Al}$ are observable in our spectra. We measured the $E W$ s of the O I permitted triplet in the near IR (7771-7774 $\AA$ ) in all program RR Lyraestars; however, these lines are very strong and their formation is likely affected by significant departures from LTE. The much weaker permitted triplet at 6155-6158 ^ was observed in the two most metal-rich stars (SW And and V445 Oph). Since these lines are rather weak, departures from LTE are expected to be small: however these lines are blended at the resolution of our spectra, so that we derived $\mathrm{O}$ abundances by comparison with synthetic spectra. The line list used in these computations includes those lines from Kurucz \& Peytremann (1975); wavelengths and $g f$ s for the stronger lines were adjusted using updated literature data and a comparison with the solar flux spectrum by Kurucz et al (1984). For these metal-rich stars, we were also able to measure $E W \mathrm{~s}$ for lines of the C I multiplet at 7111-7120 A. Finally, Na and $\mathrm{Al}$ abundances were measured from spectral synthesis of the doublet at 5682-88 $\AA$ (which unfortunately are too weak to be measured in the spectra of the most metal-poor RR Lyraestars), of the D lines, and of the $\mathrm{Al}$ resonance line at $3961 \AA$. These syntheses were obtained following the same rules adopted for the $\mathrm{O}$ lines. Figure 10 shows a comparison between synthetic and computed spectra for a few stars. Results for C, O, Na and Al abundances are summarized in Table 20. Note that $E W$ s for the O I IR triplet are more uncertain than average in our analysis, due to difficulties in eliminating the strong interference fringes present in the spectra. We 
suspect that the large O abundances for VX Her and VY Ser are due to this cause. Further, interstellar D lines are blended with stellar features in the spectra of a few of our variables, which had a low geocentric velocities when observed. No Na abundance could be drawn from the D lines when this blending was too severe (V445 Oph and SW And), while results are quite uncertain for other stars (V440 Sgr, RR Lyr, and X Ari). Uncertain values are marked by a colon in Table 20 .

Departures from LTE were considered through detailed statistical equilibrium calculations for $\mathrm{O}$ and $\mathrm{Na}$ lines, using the same procedure adopted for Fe (see Section 4.1.3). The (new) O and Na atomic models are fully described in Gratton et al (1995). As for Fe, we calibrated empirically the cross sections for collisions with H I atoms; results from these calculations are shown in Figures 11a,b. Here, we forced agreement between the abundances derived from weak and strong lines (the multiplets at 7771-74 and $6155-58 \AA$ for O; the doublets at 5893 and $5685 \AA$ for $\mathrm{Na}$ ) in the non-LTE analysis for those stars for which all these features were measured. As in Figure 8 shaded regions in Figures 11a,b correspond to the range allowed by observational errors and uncertainties in the adopted atmospheric parameters.

For Oxygen, we ran several test cases for the Sun; our results agree fairly well with those by Kiselman $(1991,1993)$. As anticipated, we found that the IR permitted triplet is much stronger than expected in LTE, while departures from LTE are negligible for the much weaker lines at $6155-58 \AA$ in the case of RR Lyraes. Our statistical equilibrium computations also show that the $\mathrm{Na} \mathrm{D}$ lines are much stronger than predicted by LTE, while, as expected, the subordinate doublet at 5682-88 $\AA$ forms close to LTE. This is due to the combination of the photon suction effect described by Bruls et al (1992), and of the depth of formation of the lines (the D lines are heavily saturated in the spectra of RR Lyrae). A more complete discussion will be given in Gratton et al (1995). We then estimated corrections to LTE abundances as a function of line strength, for model atmospheres representative of variables having different metallicities (see Figures 12a,b). The abundances listed in Table 20 include these corrections.

The results of Table 20 agree fairly well with the predictions by canonical mixing (first dredge-up: Iben 1965); all metal-poor variables have large O overabundances, while $\mathrm{Na}$ and $\mathrm{Al}$ are overdeficient with respect to $\mathrm{Fe}$. We regard as doubtful the very large $\mathrm{O}$ overabundances we found for VX Her and VY Ser. These results reproduce the typical pattern observed in less evolved metal-poor stars (see Figure 13). Our Na abundances are even slightly smaller than those observed by others, who however did not include any non-LTE correction. Therefore, we do not find any evidence for an anticorrelation between $\mathrm{Na}$ and $\mathrm{O}$ overabundances in our sample. This results is not totally unexpected; in fact no 
bona fide O-poor, Na-rich star has been found among field first ascent giants (see Sneden et al 1992). Their absence suggests that the environment (possibly in some indirect way) plays a main rôle in the mechanism causing the anomalous abundances observed in cluster stars (Langer et al 1993).

\section{DISCUSSION}

\subsection{A new calibration of the $\Delta \mathrm{S}$ index}

Our new iron abundances have been used to re-calibrate the $\Delta \mathrm{S}$ metallicity index. The newest and most complete $\Delta \mathrm{S}$ compilations for field RR Lyrae stars are those of B92 and SKK94. The two systems are virtually identical; in fact if we compare $\Delta \mathrm{S}$ values for the stars in common between the two samples (47 stars) we find that, after omitting the very discrepant UU Cet, the linear correlation between the two systems is:

$$
\Delta S_{\mathrm{B} 92}=0.97( \pm 0.03) \Delta S_{\mathrm{SKK} 94}+0.09( \pm 0.11)
$$

or

$$
\Delta S_{\mathrm{SKK} 94}=0.98( \pm 0.03) \Delta S_{\mathrm{B} 92}+0.21( \pm 0.11)
$$

To derive the $\Delta \mathrm{S}-[\mathrm{Fe} / \mathrm{H}]$ calibration we took therefore the mean values between $\Delta S_{\mathrm{B} 92}$ and $\Delta S_{\text {SKK94 }}$. Using for the $[\mathrm{Fe} / \mathrm{H}]$ values the average of our Fe I and Fe II abundances we obtained the new calibration:

$$
[\mathrm{Fe} / \mathrm{H}]=-0.217( \pm 0.015) \Delta S+0.04( \pm 0.05)
$$

which is the mean of the two least-squares solutions made by exchanging the dependent and the independent variables. The correlation coefficient is $\mathrm{r}=0.98$ with $\sigma=0.16$ (10 stars): this $\sigma$ is the accuracy of the $[\mathrm{Fe} / \mathrm{H}]$ value derived for a single star using $\Delta \mathrm{S}$ and our calibration. The new $\Delta \mathrm{S}$ vs $[\mathrm{Fe} / \mathrm{H}]$ calibration is shown in Figure 14a (solid line). Errors are 0.08 dex in $[\mathrm{Fe} / \mathrm{H}]$ and 0.7 units in $\Delta \mathrm{S}$ (assuming for both $\Delta \mathrm{S}$ systems an uncertainty of one unit in $\Delta \mathrm{S})$.

Our new calibration is steeper and has a larger zero-point than Butler's (1975; dotted line in Figure 14a). This results in a different metallicity scale in which old $[\mathrm{Fe} / \mathrm{H}]$ determinations are now stretched, so that we obtain larger abundances for metal-rich stars 
and lower abundances for metal-poor ones, the differences being almost negligible in the intermediate regime of metallicity $(\Delta S \simeq 4.7,[\mathrm{Fe} / \mathrm{H}] \sim-1.0)$. For $\Delta S=-1$ and $\Delta S=12$ we have that $\Delta[\mathrm{Fe} / \mathrm{H}]=[\mathrm{Fe} / \mathrm{H}]_{\text {our }}-[\mathrm{Fe} / \mathrm{H}]_{\text {Butl. }}=+0.33$ and -0.41 , respectively.

A more accurate calibration of the $\Delta \mathrm{S}$ index can be obtained by combining our $[\mathrm{Fe} / \mathrm{H}]$ values and those of Butler and coworkers (average of Butler 1975 and Butler \& Deming $1979)$, corrected for the systematic difference with respect to our $[\mathrm{Fe} / \mathrm{H}]$ values $(-0.18$ and -0.17 dex respectively, see Section 4.1.4). Although the corrected Butler's $[\mathrm{Fe} / \mathrm{H}]$ values are less accurate than ours, we nevertheless obtain a better distribution, particularly in the range $0<\Delta S<5$. We get a very tight relationship between $\Delta S$ and $[\mathrm{Fe} / \mathrm{H}]$ (see Figure 14b). The resulting calibration is:

$$
[\mathrm{Fe} / \mathrm{H}]=-0.204( \pm 0.012) \Delta S-0.10( \pm 0.19)
$$

which is the average relation obtained by exchanging dependent and independent variables. It should be noted that the slope of this relation agrees within the errors with that of the original Butler calibration $(-0.16 \pm 0.02$, m.e. rather than standard deviation). The improvement is due to both better $[\mathrm{Fe} / \mathrm{H}]$ values and better $\Delta S$. A steeper relation (slope of $-0.18 \pm 0.05)$ was obtained already by B92, still using Butler's $[\mathrm{Fe} / \mathrm{H}]$ but new $\Delta \mathrm{S}$. Of course, eq. (6) agrees well with eq. (5).

\subsubsection{Comparison with the Globular Cluster metallicity scale}

The new calibration of $\Delta S$ may be compared with the results obtained by using RR Lyraes in globular clusters, which have $[\mathrm{Fe} / \mathrm{H}]$ determined from high dispersion spectroscopic observations of RGB stars. We collected $[\mathrm{Fe} / \mathrm{H}]$ values from Gratton et al (1989), Leep et al (1987), Brown et al (1991, 1992), Sneden et al (1991, 1992, 1994), Kraft et al $(1992,1993)$, Peterson et al (1990), and François (1991). An inter-comparison between these different sets of data shows that the $[\mathrm{Fe} / \mathrm{H}]$ values of Gratton et al (which refer to the largest number of clusters) are in good agreement with those of Leep et al, Brown et al, and François. On the other hand, $[\mathrm{Fe} / \mathrm{H}]$ values obtained by Sneden and coworkers using large samples of stars in each cluster, observed at very high resolution and S/N, are systematically larger by $\sim 0.2$ dex than those obtained by the other authors. The reason for this systematic difference is not entirely clear; we suspect it is due to a different reduction to the solar Fe abundance. However, it is not clear what procedure should be preferable, in view of the difficulties related to the way collisional damping (which is important when solar abundances are used) is handled. Hereinafter, we simply averaged abundances obtained by 
different authors for individual clusters. We verified that none of our conclusions is modified if systematic corrections as large as 0.2 dex are applied to the various sets of $[\mathrm{Fe} / \mathrm{H}]$ for globular clusters.

We then calibrated the $\Delta S$ index using only data for globular clusters. To this purpose, we used the $\Delta S$ values collected by Costar \& Smith (1988) and references therein. The regression line between $[\mathrm{Fe} / \mathrm{H}]$ and $\Delta S$, (where the usual average between values obtained exchanging dependent and independent variables was applied), is:

$$
[\mathrm{Fe} / \mathrm{H}]=-0.168( \pm 0.016) \Delta S-0.22( \pm 0.13)
$$

The difference between the slopes of eq. (6) and (7) is significant at about the $2 \sigma$ level. Although not highly significant, this difference does call for an explanation.

A direct comparison between results for field and globular stars is given in Figure 14c, where $[\mathrm{Fe} / \mathrm{H}]$ values are plotted against $\Delta S$ both for field and globular cluster RR Lyraes. While there are no RR Lyraes in metal-rich globular clusters, the two different sets of data cannot otherwise be distinguished. This suggests that the calibrations obtained from field and cluster stars differ either simply due to random variations, or because the relation between $\Delta S$ and $[\mathrm{Fe} / \mathrm{H}]$ is not linear, and the metallicity distributions of the two groups of objects are not identical. Present data are not good enough to settle this issue: in fact, while data plotted in Figure 14c may suggest a smaller slope in the range $4<\Delta S<10$, we found that the slope obtained using all objects (field and globular cluster stars) in this range $(-0.159 \pm 0.038)$ is not statistically different from the value obtained using all objects regardless of $\Delta S$. The average calibration we obtain considering both field and cluster variables is:

$$
[\mathrm{Fe} / \mathrm{H}]=-0.194( \pm 0.011) \Delta S-0.08( \pm 0.18)
$$

which can be considered our final, adopted calibration. More, accurate abundance determinations would be welcomed.

We finally remark that a non-linear relation between $\Delta S$ and $[\mathrm{Fe} / \mathrm{H}]$ could be predicted theoretically. In fact, Manduca (1981) showed that the $\Delta S$ index is expected to saturate at low metallicities $([\mathrm{Fe} / \mathrm{H}] \sim-2.5)$, while the $[\mathrm{Ca} / \mathrm{Fe}]$ ratio is known to be lower in metal-rich stars than in metal-poor, population II stars (as discussed in Section 4.2). However, if the calibration of $\Delta S$ vs $[\mathrm{Fe} / \mathrm{H}]$ is indeed affected by the trend of the $[\mathrm{Ca} / \mathrm{Fe}]$ ratio with $[\mathrm{Fe} / \mathrm{H}]$, considerable care should be devoted in extending the calibration inferred from stars in the solar neighborhood to RR Lyraes observed in environments where the run of the $\alpha$-elements with respect to Fe is expected to be different, like the galactic bulge or the Magellanic Clouds. 


\subsection{A new calibration of the $\mathrm{W}^{\prime}(\mathrm{K})$ index}

The other technique to derive the metallicity from low-resolution spectra of RR Lyrae stars is the equivalent width of the Ca II K line corrected for interstellar contribution (Clementini et al 1991). Using the present new metallicities, and $W^{\prime}(K)$ values and relative errors from Clementini et al (1991), (we have 8 objects in common with that paper, but have omitted VY Ser, whose $W^{\prime}(K)$ value is rather uncertain), a least-squares fit weighted both in $W^{\prime}(K)$ and $[\mathrm{Fe} / \mathrm{H}]$ gives:

$$
[\mathrm{Fe} / \mathrm{H}]=0.65( \pm 0.17) W^{\prime}(K)-3.49( \pm 0.39) \quad(9) \quad(7 \text { objects })
$$

where, according to the procedure by Clementini et al $(1991),[\mathrm{Fe} / \mathrm{H}]$ values were derived from Fe II abundances. The new $W^{\prime}(K)$ vs $[\mathrm{Fe} / \mathrm{H}]$ calibration is shown in Figure 15 (solid line). Error bars of the $[\mathrm{Fe} / \mathrm{H}]$ values correspond to 0.09 dex (see Section 4.1.2).

Same as for $\Delta \mathrm{S}$, the new $W^{\prime}(K)$ vs $[\mathrm{Fe} / \mathrm{H}]$ calibration is steeper and has a larger zero-point than Clementini's et al (1991; dotted line in Figure 15). This translates in a larger metal abundance derived for metal-rich RR Lyraes and lower abundances for metal-poor ones. For $W^{\prime}(K)=1$ and $W^{\prime}(K)=6.5$ we have that $\Delta[\mathrm{Fe} / \mathrm{H}]=[\mathrm{Fe} / \mathrm{H}]_{\text {our }}-[\mathrm{Fe} / \mathrm{H}]_{\mathrm{Clem}}=-0.30$ and +0.31 , respectively, while we get almost the same metallicity $[\mathrm{Fe} / \mathrm{H}] \sim-1.2$, for $W^{\prime}(K)=3.5$.

\subsection{The $\mathrm{M}_{V}-[\mathrm{Fe} / \mathrm{H}]$ dependence}

We have used our new metallicity scale to revise the metallicity dependence of the absolute magnitude of RR Lyraestars, $\mathrm{M}_{V}$. This is a very crucial step in deriving the galactic distance ladder and the ages of Galactic Globular Clusters (GGCs). If accurately known, the slope of this relationship could allow us to calibrate the relative ages of GGCs, discriminating between the slow and rapid halo collapse scenarios, while the zero-point would fix the age of some reference cluster, enabling absolute age determinations.

Our aim is to see if the new metallicity scale has some effect on the slope of $\mathrm{M}_{V}-$ $[\mathrm{Fe} / \mathrm{H}]$ dependence. The most up-dated list of $\mathrm{B}-\mathrm{W} \mathrm{M}_{V}$ estimates derived from infrared colors was published by Fernley (1994), who also provided a re-evaluation of the $\mathrm{M}_{V}$ values using a perhaps more appropriate value of the conversion factor between observed and true pulsation velocity $p=1.38$ than previously adopted (see Fernley 1994 for a detailed discussion of this issue). Fernley's (1994) sample contains 29 field RR Lyraes. To these, 
we added 8 globular cluster variables on which BW analysis has been performed by Liu \& Janes (1990b; V2, V15, V32, V33 in M4), and by Storm et al (1994; V8 and V28 in M5, and V1 and V3 in M92). The relevant data for these stars are collected in Table 21 where we give the star name (Column 1); $\Delta$ S values obtained as the average between B92 and SKK94 values (Column 2); $[\mathrm{Fe} / \mathrm{H}]$ values and related uncertainties (Column 3 and 4 respectively), from high resolution abundance analysis or derived from the $\Delta \mathrm{S}$ values in Column 2 and our $\Delta \mathrm{S}-[\mathrm{Fe} / \mathrm{H}]$ calibration (eq. 8); the absolute magnitudes $\mathrm{M}_{V}$ from Fernley (1994) for the field stars, listing both the original values (Column 5 ) and the values corrected to $p=1.38$ (Column 6), and the associated errors (Column 7: from Skillen et al 1993).

For the cluster variables, $\mathrm{M}_{V}$ values in Column 5 and uncertainties in Column 7 are from Liu \& Janes (1990b), and Storm et al (1994), respectively. These $\mathrm{M}_{V} \mathrm{~s}$ were derived using $p=1.31$ and 1.30. According to Table 1 of Fernley (1994) the $\mathrm{M}_{V}$ values of the M4 variables were made brighter by $0.11 \mathrm{mag}$ and those of M5 and M92 were made brighter by $0.13 \mathrm{mag}$ to transform them to $p=1.38$ (see values in Column 6). Metallicity for the M4 variables is the average value derived in Paper I. For M5 and M92 we used $[\mathrm{Fe} / \mathrm{H}]=-1.17$ and $[\mathrm{Fe} / \mathrm{H}]=-2.25$, as derived by Sneden et al $(1994,1992)$ from high resolution spectroscopy of RGB stars. The adoption of a metallicity slightly different from that used to derive the $\mathrm{M}_{V}$ values via $\mathrm{B}-\mathrm{W}$ analysis should not affect our conclusions since all the quoted $\mathrm{B}-\mathrm{W} \mathrm{M}_{V}$ estimates were obtained by using the $V-K$ color that is almost metallicity independent.

The data listed in Table 21 are plotted in Figure 16, with the original $\mathrm{M}_{V}$ values in panel (a) and the value corrected to $p=1.38$ in panel (b). Different symbols in Figure 16 refer to: field RR Lyraes (open and filled squares, the last ones being the objects studied in the present paper, for them, we have used our [Fe/H] estimates); RR Lyraes in M5 and M92 (open triangles and open circles, respectively) according to Storm et al (1994); and RR Lyraes in M4 (filled triangles) according to Liu \& Janes (1991). Asterisks mark field variables that are claimed to be evolved by Jones et al (1992), Cacciari et al (1992), and Skillen et al (1993). We note that, a part from SS Leo and the two variables in M92, all the other stars do not seem to deviate significantly from the general distribution in panels (a) and (b). Moreover BB Pup is underluminous, while evolution off the ZAHB should make the star overluminous.

Omitting SS Leo and the M92 stars, (see the discussion on the reasons for omitting evolved stars when calculating the $\mathrm{M}_{V}$ vs $[\mathrm{Fe} / \mathrm{H}]$ relation in Cacciari et al 1992), a least-squares fit weighted both in $\mathrm{M}_{V}$ and $[\mathrm{Fe} / \mathrm{H}]$ gives:

$$
\mathrm{M}_{V}=0.20( \pm 0.03)[\mathrm{Fe} / \mathrm{H}]+1.06( \pm 0.04) \quad(r=0.72 \quad 34 \text { objects })
$$


which was obtained using the $\mathrm{M}_{V}$ values in Column 5 of Table 21, and:

$$
\mathrm{M}_{V}=0.19( \pm 0.03)[\mathrm{Fe} / \mathrm{H}]+0.96( \pm 0.04) \quad(r=0.70 \quad 34 \text { objects })
$$

which was obtained using the corrected $\mathrm{M}_{V}$ values in Column 6 of Table 21. These best-fit regression lines are plotted on the data in Figure 16.

Analogous best-fit calculations, but omitting all the supposed evolved stars, (SU Dra, W Tuc, SS Leo, BB Pup and the M92 variables) give:

$$
\mathrm{M}_{V}=0.17( \pm 0.03)[\mathrm{Fe} / \mathrm{H}]+1.03( \pm 0.04) \quad(r=0.76 \quad 31 \text { objects }) \quad(10 \mathrm{c})
$$

and

$$
\mathrm{M}_{V}=0.17( \pm 0.03)[\mathrm{Fe} / \mathrm{H}]+0.93( \pm 0.04) \quad(r=0.73 \quad 31 \text { objects }) \quad(10 \mathrm{~d})
$$

respectively. The adoption of the new metallicity scale does not yield significant changes in the slope and zero-point of the $\mathrm{M}_{V}$ vs $[\mathrm{Fe} / \mathrm{H}]$ relation. Indeed, within the quoted uncertainties, slopes and zero-points of eq. 10a and 10c agree very well with those found by Liu \& Janes (1990a,b), Jones et al (1992), Cacciari et al (1992), and Skillen et al (1993); therefore the conclusions drawn in those papers, in particular about the ages of the Galactic globular clusters, apply here as well. According to Fernley (1994), a zero-point brighter by $0.10 \mathrm{mag}$ is found when using $\mathrm{M}_{V}$ values derived for $p=1.38$ (see eq. $10 \mathrm{~b}$ and $10 \mathrm{~d}$ ). However this zero-point is still $\sim 0.08 \mathrm{mag}$ fainter than the zero-points of the $\mathrm{M}_{V}$ vs $[\mathrm{Fe} / \mathrm{H}]$ relations predicted by Stellar Evolution and Stellar Pulsation theories (Fernley 1994).

On the other hand, the distributions in Fig.s 16a,b, do not seem to be well fitted by a single-sloped relationship. Once evolved stars are eliminated, it appears that there might be two distinct regimes: one, at high metallicity, requiring a higher slope, and a second one at low metallicity, which would better be fitted with a much lower slope. If true, this result would not be entirely unexpected, since Castellani et al (1991) have already pointed out (from a theoretical point of view) that there is no reason, in principle, to believe in a linear dependence of the HB luminosities on metallicity. Their point is that at lower metallicities this dependence is much smaller than in the higher metallicities regime, where the theoretical slope rises progressively, approaching the Sandage's (1993a,b) pulsational value. Our analysis seems to give some support to this prediction; however, a much larger data sample (particularly $\mathrm{B}-\mathrm{W} \mathrm{M}_{V}$ determinations) are requested to definitely settle this question.

\section{CONCLUSIONS}


We derived abundances for 21 species from moderately high-resolution, high $\mathrm{S} / \mathrm{N}$, visible spectra of 10 field $a b$-type RR Lyraestars. The main purposes of this study were:

- to provide new, updated calibrations of the $\Delta \mathrm{S}$ (Preston 1959) and Ca II K line (Clementini et al 1991) indices to be used in galactic and extragalactic studies.

- to discuss the composition of HB stars, comparing it to that of less evolved low-mass stars.

- to compare the observed line strengths with predictions from statistical equilibrium calculations, in order to predict the relevance of departures from LTE in the spectra of subdwarfs and RGB stars, where non-LTE effects are expected to be smaller than for RR Lyraes.

In order to achieve these results, we put particular emphasis on a precise estimate of the atmospheric parameters independently of excitation and ionization equilibria. To this purpose, we selected stars having accurate photometric and radial velocity data, and made observations close to minimum phase, when the atmospheres are quite stable and shock waves are out of the line forming regions. Photometric reddening estimates for the program stars were carefully examined, and compared with other determinations. Dereddened colors were then used to infer effective temperatures at the phase of observations using a new temperature scale determined from literature Infrared Flux Method measures of subdwarfs, and the Kurucz (1992) model atmospheres, following a procedure similar to that recently adopted by King (1993). Gravities were derived from the radial velocity curve of the variables. The applicability of Kurucz (1992) model atmospheres in the analysis of RR Lyraes at minimum light was briefly analyzed: we found that they are able to reproduce colors, excitation and ionization equilibria as well as the wings of $\mathrm{H}_{\alpha}$. The solar comparison abundances were carefully determined. In particular, a new solar Fe abundance was obtained from a group of weak Fe I lines having accurate $g f_{\mathrm{s}}$ (Bard \& Kock 1994). We obtained a value of $\log \epsilon(F e)=7.52$, in good agreement with the value obtained from meteorites and Fe I lines.

The main results of our abundance analysis for RR Lyraes are:

- The metal abundances of the program stars span the range $-2.50<[\mathrm{Fe} / \mathrm{H}]<+0.17$.

- Lines of most elements are found to form in LTE conditions; there are a few significant exceptions, that are mentioned below. In particular, we found that the equilibria of excitation and ionization are very well achieved for Fe lines. This result is compared with predictions from statistical equilibrium calculations, using a rather extended Fe I 
model atom, including 60 levels plus the continuum. We found that the originally adopted collisional cross sections must be raised in order to reproduce observations. If statistical equilibrium computations with this rather large collisional cross sections are then repeated for subdwarfs and metal-poor giants, departures from LTE are found to be negligible, thus validating the LTE analyses for these stars.

- On the whole, the composition of RR Lyraes is similar to that of less evolved stars of similar $[\mathrm{Fe} / \mathrm{H}]: \alpha$-elements are overabundant by $\sim 0.4$ dex and $\mathrm{Mn}$ is underabundant by $\sim 0.6$ dex in stars with $[\mathrm{Fe} / \mathrm{H}]<-1$. Solar scaled abundances are found for most of the other species, except for the low Ba abundance in the extremely metal-poor star $\mathrm{X}$ Ari $([\mathrm{Fe} / \mathrm{H}] \sim-2.5)$.

- Significant departures from LTE are found for a few species: Nd II, Ce II, Y II and Sc II are severely underabundant $(\sim 0.5 \mathrm{dex})$ in metal-rich variables; Ti I and $\mathrm{Cr}$ I are slightly $(\sim 0.1-0.2 \mathrm{dex})$ underabundant in metal-poor stars. These effects are attributed to overionization. We suggest that the photoionization of the alkaline earth-like ions is due to emission in the Lyman lines produced by shock waves that propagate in the atmosphere of these variables (Fokin 1992). However, it is not clear why this effect is not seen in more metal-poor stars, and it is not observed for Ca and Ba.

- Departures from LTE were considered in detail in the derivation of abundances for the light elements $(\mathrm{O}$ and $\mathrm{Na}$ ). A comparison with weaker lines (forming close to LTE) showed that significant corrections were required for the O I IR triplet and the $\mathrm{Na} \mathrm{D}$ lines. We were able to model these non-LTE effects by means of statistical equilibrium computations. The abundances obtained from the non-LTE analysis reproduce very well those observed in less evolved field stars, suggesting that the mixing during the RGB phase did not involve ON-processed material for all program stars. Hence, we did not find any evidence for an O-Na anti-correlation among these field HB-stars, suggesting that the environment is likely to be somehow responsible for the anti-correlation found in metal-poor globular cluster stars (Sneden et al 1992).

A new calibration of the $\Delta \mathrm{S}$ index for $a b$-type $\mathrm{RR}$ Lyraes was then obtained using our own $[\mathrm{Fe} / \mathrm{H}]$, as well as those from Butler and coworkers (corrected to our system), and from high resolution spectroscopy of globular clusters giants:

$$
[\mathrm{Fe} / \mathrm{H}]=-0.194( \pm 0.011) \Delta S-0.08( \pm 0.18)
$$

Our new metallicity scale is stretched on both low and high metallicity ends with respect to Butler's (1975). There is some hint that $\Delta \mathrm{S}-[\mathrm{Fe} / \mathrm{H}]$ relation may be not linear: in fact 
the slope obtained using only stars with $4<\Delta S<10$ is slightly smaller than that obtained using all stars. While this difference is only barely significant, it would not be in contrast with theoretical expectations. In fact, a saturation of the $\Delta S$ index at low metallicities has been suggested by Manduca (1981), while at high metallicities we expect a larger slope due to the variation of the $[\mathrm{Ca} / \mathrm{Fe}]$ ratio with $[\mathrm{Fe} / \mathrm{H}]$ in the range $-1<[\mathrm{Fe} / \mathrm{H}]<0$. If this non linearity is confirmed by more observations, caution should be exerted in the derivation of $[\mathrm{Fe} / \mathrm{H}]$ from $\Delta \mathrm{S}$ for stars having $[\mathrm{Ca} / \mathrm{Fe}]$ ratios different from that observed in stars of similar $[\mathrm{Fe} / \mathrm{H}]$ in the solar neighborhood.

In a similar way, we used our new $[\mathrm{Fe} / \mathrm{H}]$ values to update the metallicity calibration of the Ca II K lineindex. Using the present new metallicities, and $W^{\prime}(K)$ values and relative errors from Clementini et al (1991), a least-squares fit weighted both in $W^{\prime}(K)$ and $[\mathrm{Fe} / \mathrm{H}]$ gives:

$$
[\mathrm{Fe} / \mathrm{H}]=0.65( \pm 0.17) W^{\prime}(K)-3.49( \pm 0.39)
$$

Finally, our new metallicity scale was used to revise the metallicity dependence of the absolute magnitude of RR Lyraestars, $\mathrm{M}_{V}$. We found:

$$
\mathrm{M}_{V}=0.20( \pm 0.03)[\mathrm{Fe} / \mathrm{H}]+1.06( \pm 0.04)
$$

and:

$$
\mathrm{M}_{V}=0.19( \pm 0.03)[\mathrm{Fe} / \mathrm{H}]+0.96( \pm 0.04)
$$

the last being obtained by using $\mathrm{M}_{V}$ values derived for a value of the conversion factor between observed and true pulsation velocity $p=1.38$ (Fernley 1994). As expected, the adoption of the new metallicity scale does not yield significant changes in the slope and zero-point of the $\mathrm{M}_{V}$ vs $[\mathrm{Fe} / \mathrm{H}]$ relation. A close inspection of the available data does not exclude the possibility that there might be two distinct regimes in the $\mathrm{M}_{V}$ vs $[\mathrm{Fe} / \mathrm{H}] \mathrm{plane}$ : one, at high metallicity, requiring a higher slope, and a second one at low metallicity, which would better be fitted with a much lower slope. A larger data sample (particularly B-W $\mathrm{M}_{V}$ determinations) would be requested, however, to definitely settle this question.

\section{Acknowledgments}

We wish to thank Dr. R.L. Kurucz for kindly making available to us on tape his new model atmospheres; Dr. M. Carlsson for having provided a version of the MULTI code for statistical equilibrium computations; Dr. C. Cacciari for her comments on the color- $T_{\text {eff }}$ calibration; Prof. B. Gustafsson for many comments on statistical equilibrium computations; and Dr. A. Bragaglia for help in the reduction procedures and preparation 
of figures. We also thank the referee, Dr. R. Peterson, for making constructive suggestions concerning the original manuscript. G.C. and R.G. wish to dedicate this paper to their children Ilaria and Max.

\section{Appendix}

In order to derive temperatures from observed colors, we have collected from the literature the most recent photometric data available for our program stars. In the following we list the photometric data used for each star, giving also the equations used to transform original photometries to the Johnson-Cousins $\left(B V R_{C} I_{C} K_{J}\right)$ photometric system.

$-\mathrm{RR}$ Cet

$B V R I$ on the Johnson-Cousins system $\left(B V R_{C} I_{C}\right)$, and $K$ photometry on the CIT system $\left(K_{C I T}\right)$ transformed to Johnson according to eq. (a) (see below), published by Liu \& Janes (1989).

-RR Lyr

$B V$ from Fitch et al (1966), $V$ from Siegel (1982), and $B V R I$ from Manduca et al (1981). Manduca et al (1981) photometry is on the Johnson system defined by Barnes et al (1978) $\left(R_{B M}, I_{B M}\right)$, and was transformed to Cousins using eq. (b) (see below).

-VY Ser

V photometry from Carney \& Latham (1984), $\mathrm{K}_{C I T}$ photometry from Jones et al (1988), $\mathrm{R}_{B M}$ and $\mathrm{I}_{B M}$ from Fernley et al (1990), transformed to Johnson-Cousins using eqs. (a) and (b).

-X Ari

$V R_{C} K_{C I T}$ from Jones et al (1987), $B V$ from Burchi et al (1993), and $B V R_{C} I_{C} K_{J}$ from Fernley et al (1989) as derived from their Figures 1 and 2 which include also the $V R$ photometry originally taken from Manduca et al (1981).

-ST Boo

$B V R I$ from Clementini et al (1995). $R$ and $I$ data are on the Johnson system defined by Nechel \& Chini (1980) $\left(R_{N C}, I_{N C}\right)$; they were transformed to Cousins using eq. (c).

-UU Cet

$B V R_{C} I_{C}$ photometry from Clementini et al (1990), and $K$ photometry on the ESO photometric system $\left(K_{E S O}\right)$ from Cacciari et al (1992). $K_{E S O}$ has been transformed to $K_{J}$ using eq. (d).

$-\mathrm{V} 445 \mathrm{Oph}$ 
$V K_{J}$ from Fernley et al (1990), and $B V R_{B M} I_{B M}$ from Barnes et al (1988), transformed according to eq. (b).

-V440 Sgr

$B V R_{C} I_{C}$ from Cacciari et al (1987).

-VX Her

$B V$ photometry from Stepien (1972), Fitch et al (1966), Sturch (1966) and $B V R_{N C} I_{N C}$ from Clementini (unpublished), transformed according to eq. (c).

We have used the following equations to transform original photometries to the JohnsonCousins system:

$$
\begin{aligned}
& (V-K)_{J}=(V-K)_{C I T}-0.011 \quad(\text { Jones et al 1987) (a) } \\
& (V-R)_{C}=0.716(V-R)_{B M}-0.032 \quad(R-I)_{C}=0.873(R-I)_{B M}+0.42 \quad \text { Bessel (1983) (b) } \\
& (V-R)_{C}=0.757(V-R)_{N C}-0.040 \quad(V-I)_{C}=0.782(V-I)_{N C} \quad \text { Bessel (1983) (c) } \\
& (V-K)_{J}=(V-K)_{E S O}-0.013-0.024(J-K)_{E S O} \quad \text { Cacciari et al (1992) (d) }
\end{aligned}
$$




\section{REFERENCES}

Alania, I.F. 1973, Byull. Abastumansk. Astrofiz. Obs., 45, 3

Ali, A.W., Griem, H.R. 1965, Phys. Rev., 140, 1044

Ali, A.W., Griem, H.R. 1966, Phys. Rev., 144, 366

Anders, E., \& Grevesse, N. 1990, Geochim. Cosmochim. Acta, 53, 197

Bard, A., \& Kock, M. 1994, A\&A, 282, 1014

Bard, A., Kock, A., \& Kock, M. 1991, A\&A, 248, 315

Barnes, T.G., Evans, D.S., \& Moffett, T.J. 1978, MNRAS, 183, 285

Barnes, T.G., Moffett, T.J., Hawley, S.L., Slovak, M.H., and Frueh, M.L. 1988, ApJS, 67, 403

Benetti, S., Patat, F., Turatto, M., Contarini, G., Gratton, R.G., \& Cappellaro, E. 1994, A\&A, 285, L13

Bessell, M.S. 1979, PASP, 91, 589

Bessell, M.S. 1983, PASP, 95, 480

Biémont, E., Baudoux, M., Kurucz, R.L., Ansbacher, W., \& Pinnington, E.H. 1991, A\&A, 249,539

Blackwell, D.E., \& Lynas-Gray A.E. 1994, A\&A, 282, 899 (BLG94)

Blackwell, D.E., \& Shallis, M.J. 1977, MNRAS, 180, 177

Blackwell, D.E., Shallis, M.J., \& Simmons, G.J. 1980, A\&A, 81, 340

Blanco, V.M. 1992, AJ, 104, 734 (B92)

Brown, J.A., \& Wallerstein, G. 1992, AJ, 104, 1818

Brown, J.A., Wallerstein, G., \& Oke, J.B. 1991, AJ, 101, 1693

Bruls, J.H.N.J., Rutten, R.J., Shchukina, N.G. 1992, A\&A, 265, 237

Burchi, R., De Santis, R., Di Paoloantonio, A., \& Piersimoni, A.M. 1993, A\&AS, 97, 827

Burstein, D., \& Heiles, C. 1978, ApJ, 225, 40 (BH78)

Burstein, D., \& Heiles, C. 1982, AJ, 87, 1165 (BH82)

Butler, D. 1975, ApJ, 200, 68

Butler, D. \& Deming, D. 1979, AJ, 84, 86

Cacciari, C., Clementini, G., \& Fernley, J.A. 1992, AJ, 396, 219 
Cacciari, C., Clementini, G., Prévot, L., Lolli, M., \& Oculi, L. 1987, A\&AS, 69, 135

Cacciari, C., Clementini, G., Prévot, L., \& Buser, R. 1989a, A\&A, 209, 141

Cacciari, C., Clementini, G., \& Buser, R. 1989b, A\&A, 209, 154

Caccin, B., Gomez, M.T., \& Severino, G. 1993, A\&A, 276, 219

Cardelli, J.A., Clayton, G.C., \& Mathis, J.S. 1989, ApJ, 345, 245

Carney, B.W., \& Jones, R. 1983, PASP, 95, 246

Carney, B.W., \& Latham, D.W. 1984, ApJ, 278, 241

Carretta, E., \& Gratton, R.G. 1992, in 3rd DAEC Meeting on The feedback of chemical evolution on the stellar content of galaxies, Eds. D. Alloin and G. Stasinka, Pub. Obs. Paris, p.207

Carretta, E., Gratton, R.G., \& Sneden, C. 1995, in preparation

Castellani, V., Chieffi, A., \& Pulone, L. 1991, ApJS, 76, 911

Clementini, G., Cacciari, C., \& Lindgren, H. 1990, A\&AS, 85, 865

Clementini, G., Tosi, M., \& Merighi, R. 1991, AJ, 101,2168

Clementini, G., Merighi, R., Gratton, R., \& Carretta, E. 1994a, MNRAS, 267, 43 (Paper I)

Clementini, G., Merighi, R., Pasquini, L., Cacciari, C., \& Gouiffes, C. 1994b, MNRAS, 267, 83

Clementini, G., Cacciari, C., Prevot, L., \& Lolli, M., 1995, in preparation

Clube, S.V.M., Evans, D.S., \& Jones, D.H.P. 1969, MNRAS, 72, 101

Costar, D. \& Smith, H.A. 1988, AJ, 96, 1925

Cousins, A.W.J. 1980, SAAO Circ. 1, 234

Denisenkov, P.A., \& Denisenkova, S.N. 1990, Sov. Astron. Lett, 16, 275

Drake, J., Plez, B., \& Smith, V.V. 1993, ApJ, 412, 612

Drake, J., Smith, V.V., \& Suntzeff, N.B. 1992, ApJ, 395, L95

Drawin, H.W. 1968, Z. f. Physik, 211, 404

Drawin, H.W. 1969, Z. f. Physik, 225, 470

Edvardsson, B., Andersen, J., Gustafsson, B., Lambert, D.L., Nissen, P.E., \& Tomkin, K. 1993, A\&A, 275, 101

Fernley, J.A. 1989, MNRAS, 239, 905 (F89)

Fernley, J.A., Lynas-Gray, A.E., Skillen, I., Jameson, R.F., Marang, F., Kilkenny, D., \& Lomgmore, A.J. 1989, MNRAS, 236, 447 
Fernley, J.A., Skillen, I., Jameson, R.F., Barnes, T.G., Kilkenny, D., \& Hill, G. 1990, MNRAS, 247, 287

Fernley, J.A. 1994, A\&A Letters, 284, L16

Fitch, W.S., Wisniewski, W.Z., \& Johnson, H.L. 1966, Communs. Lunar Planet. Lab., 71, 3

Fokin, A.B. 1992, MNRAS, 256, 26

François, P. 1991, A\&A, 247, 56

Fuhrmann, K., Axer, M., Gehren, T. 1993, A\&A, 271, 462

Fuhrmann, K., Axer, M., Gehren, T. 1994, A\&A, 285, 585

Gillet, D., \& Crowe, R.A. 1988, A\&A, 199, 242

Gratton, R.G. 1987, in Stellar Evolution and Dynamics in the Outer Halo of the Galaxy, eds. M. Azzopardi \& F. Matteucci (Garching: ESO), p. 153

Gratton, R.G. 1988, Rome Obs. Preprint Ser. 29

Gratton, R.G. 1989, A\&A, 208, 171

Gratton, R.G., \& Ortolani, S. 1989, A\&A, 211, 41

Gratton, R.G., \& Sneden, C. 1991, A\&A, 241, 501

Gratton, R.G., \& Sneden, C. 1994, A\&A, 287, 927

Gratton, R.G., Carretta, E., Gustafsson, B., \& Eriksson, K. 1995, in preparation

Gustafsson, B., Bell, R.A., Eriksson, K., \& Nordlund, A. 1975, A\&A, 42, 407

Hannaford, P., Lowe, R.M., Grevesse, N., \& Noels, A. 1992, A\&A, 259, 301

Heise, C., \& Kock, M. 1990, A\&A, 230, 244

Herbig, G.H. 1993, ApJ, 407, 142

Holweger, H., \& Müller E.A. 1974, Solar Phys., 39, 19 (HM)

Holweger, H., Heise, C., \& Kock, M. 1990, A\&A, 232, 510

Holweger, H., Bard, A., Kock, A., \& Kock, M. 1991, A\&A, 249, 545

Howarth, I.D. 1983, MNRAS, 203, 301

Iben, I.Jr. 1965, ApJ, 142, 1447

Jones, D.H.P. 1973, ApJS, 25, 487

Jones, R.V. 1987, Ph.D. Thesis, University of North Carolina, Chapel Hill

Jones, R.V., Carney, B.W., \& Latham, D.W. 1988, ApJ, 332, 206 
Jones, R.V., Carney, B.W., Latham, D.W., \& Kurucz, R.l. 1987, ApJ, 312, 254

Jones, R.V., Carney, B.W., Storm, J., \& Latham, D.W. 1992, ApJ, 386, 646

Kaulakys, B. 1985, J. Sov. Phys. B: At. Mol. Phys., 18, L167

Kaulakys, B. 1986, Sov. Phys. JEPT 64, 229

King, J.R. 1993, AJ, 106, 1206

Kinman, T.D., \& Carretta, E. 1992, PASP, 104,111

Kiselman, D. 1991, A\&A, 245, L9

Kiselman, D. 1993, A\&A, 275, 269

Kraft, R.P., Sneden, C., Langer, G.E., \& Prosser, C.F. 1992, AJ, 104, 645

Kraft, R.P., Sneden, C., Langer, G.E., \& Shetrone, M.D. 1993, AJ, 106, 1490

Kurucz, R.L. 1979, ApJS, 40, 1 (K79)

Kurucz, R.L., \& Peytremann, E. 1975, SAO Special Rep. 362

Kurucz, R.L., Furenlid, I., Brault, J., \& Testerman, L. 1984, Solar Flux Atlas from 296 to $1300 \mathrm{~nm}$ (Harvard, Cambridge)

Kurucz, R.L. 1992, private communication (K92)

Langer, G.E., Hoffman, R., \& Sneden, C. 1993, PASP, 105, 301

Leep, E., Oke, J.B., \& Wallerstein, G. 1987, AJ, 92, 388

Liu, T., \& Janes, K.A. 1989, ApJS, 69, 593

Liu, T., \& Janes, K.A. 1990a, ApJ, 354, 273

Liu,T. \& Janes, K.A. 1990b, ApJ, 360, 561

Lub, J. 1977a, A\&AS, 29, 345

Lub, J. 1977b, Ph.D. thesis, University of Leiden

Lub, J. 1979, AJ, 84, 383

Luck, R., \& Bond, H.E. 1989, ApJS, 71, 559

Luck, R., Bond, H.E., \& Lambert, D.L. 1990, ApJ, 357, 188

McCarthy, J.K. 1988, Ph.D. thesis, California Institute of Technology

McDonald, L.H. 1977, Ph.D. thesis, University of California, Santa Cruz

Magain, P. 1986, A\&A, 163, 135

Magain, P. 1989, A\&A, 209, 211 
Maltby, P., Avrett, E.H., Carlsson, M., Kjeldseth-Moe, O., Kurucz, R.L., \& Loeser, R., 1986, ApJ, 306, 284

Manduca, A. 1981, ApJ, 245, 258

Manduca, A., Bell, R.A., Barnes, T.G.III, Moffett, T.J., \& Evans, D.S. 1981, ApJ, 250, 312

Moore, C.E., Minnaert, M.G.J., Houtgast, J. 1966, The Solar Spectrum 2935 Ato $8770 \AA$ A, NBS Mono. 61

Nechel, Th., \& Chini, R. 1980, A\&AS, 39, 411

Nissen, P.E., Gustafsson, B., Edvardsson, B., \& Gilmore, G. 1994, A\&A, 285, 440

O’Brian, T.R., Wickliffe, M.E., Lawler, J.E., Whaling, W., \& Brault, J.W. 1991, JOSA, B8, 1185

Oke, J.B. 1966, ApJ, 145, 468

Peterson, R.C., Kurucz, R.L., \& Carney, B.W. 1990, ApJ, 350, 173

Preston, G.W. 1959, ApJ, 130, 507

Preston, G.W., \& Paczynski B. 1964, ApJ, 140, 181

Pritchet, C.S., \& van den Bergh, S. 1987, ApJ, 316, 517

Ringe, P. 1985, BAV Rundbrief, 34, 1

Rutten, R.J., \& van der Zalm, E.B.J. 1984, A\&AS, 55, 143

Saha, A., Hoessel, J.G., \& Krist, J. 1992a, AJ, 103, 84

Saha, A., Freedman, W.L., Hoessel, J.G., \& Mossman, A.E. 1992b, AJ, 104, 1072

Sandage, A. 1993a, AJ, 106, 703

Sandage, A. 1993b, AJ, 106, 719

Sanford, R. 1949, ApJ, 109, 208

Saxner, M. \& Hammarbäck, G. 1985, A\&A, 151, 372 (SH85)

Scharmer, G.B., \& Carlsson, M. 1985, J. Comput. Phys., 59, 56

Siegel, M.J. 1982, PASP, 94, 122

Simmons, G.J. \& Blackwell, D.E. 1982, A\&A, 112, 209

Skillen, I., Fernley, J.A., Stobie, R.S., \& Jameson, R.F. 1993, MNRAS, 265, 301

Smith, H.A 1984, ApJ, 281, 148

Smith, H.A 1990, PASP, 102, 124

Smith, H.A, \& Butler, D. 1978, PASP, 90, 671 
Sneden, C., Kraft, R.P., Langer, G.E., Prosser, C.F., \& Shetrone, M.D. 1994, AJ, 107, 1773

Sneden, C., Kraft, R.P., Prosser, C.F., \& Langer, G.E. 1991, AJ, 102, 2001

Sneden, C., Kraft, R.P., Prosser, C.F., \& Langer, G.E. 1992, AJ, 104, 2121

Sneden, C., Gratton, R.G., \& Crocker, D.A. 1991, A\&A, 246, 354

Steenbock, W. 1985, in Cool Stars with Excess of Heavy Elements, eds. M. Jaschek \& P.C. Keenan (Dordrecht: Reidel), p. 231

Steenbock, W., \& Holweger, H. 1984, A\&A, 130, 319

Stepien, K. 1972, Acta Astron., 22, 175

Storm, J., Carney, B., W., \& Latham, D.W. 1994, A\&A, 290, 443

Sturch, C. 1966, ApJ, 143, 774

Suntzeff, N.B., Kinman, T.D., \& Kraft, R.P. 1991, ApJ, 367, 528, (SKK91)

Suntzeff, N.B., Kraft, R.P., \& Kinman, T.D. 1994, ApJS, 93, 271 (SKK94)

Sweigart, A.V., \& Mengel, J.G. 1979, ApJ, 229, 624

Tassoul, M., \& Tassoul, J.-L. 1984, ApJ, 279, 384

Vidal, C.R., Cooper, J., Smith, E.W. 1970, JQSRT, 10, 1011

Walker, A.R. 1991, In The Magellanic Clouds, ed. R.Haynes \& D.Milne (Boston: Kluwer), 307

Walker, A.R., \& Terndrup, D.M. 1991, ApJ, 378, 119

Woolley, R., \& Savage, A. 1971, Royal Obs. Bull., 170, 365

Zhao, G., \& Magain, P. 1990, A\&A, 238, 242 


\section{Figure captions}

Figure 1. Tracing of a portion of the co-added normalized spectrum of X Ari, RR Cet and SW And (upper, middle and lower tracing, respectively). The spectra of X Ari and RR Cet were arbitrarily offset vertically to avoid overposition of the plots.

Figure 2. Comparison between measured $E W$ s of RR Lyr and RR Cet. The best-fit regression line with zero constant value is overposed (see text).

Figure 3. Comparison between theoretical K92 and empirical color- $T_{\text {eff }}$ calibrations (dashed and solid lines, respectively). Filled circles are the Population I main sequence stars listed in Table 7.

Figure 4. Comparisons between observed $\mathrm{H}_{\alpha}$ profiles for $\mathrm{X}$ Ari, RR Cet, and SW And (thick lines), and synthetic spectra computed using K92 model atmospheres with $T_{\text {eff }}$ of $5500,5750,6000,6250$, and $6500 \mathrm{~K}$ (thin lines: $\mathrm{H}_{\alpha}$ becomes stronger with increasing $T_{\text {eff }}$ ). The spectra of RR Cet and SW And were arbitrarily offset vertically to avoid overposition of the plots. Synthesized profiles were computed assuming a surface gravity of $\log g=2.75$ and a metal abundance of $[\mathrm{A} / \mathrm{H}]=-1 . T_{\text {eff }}$ 's derived from these fits were corrected downward by $247 \mathrm{~K}$, to account for the systematic correction determined from an analogous fit of the solar $\mathrm{H}_{\alpha}$ profile.

Figure 5. Comparison between the temperature stratification in the atmosphere of a typical RR Lyraestar at minimum light as derived from K79 and K92 models, respectively.

Figure 6. (a) Plot of the residuals of abundances deduced from individual Fe lines minus the average Fe abundances for each star, against $E W \mathrm{~s}$, for all program stars; (b) Plot of the average residuals of panel $6 \mathrm{a}$, calculated in bins of 0.2 dex, against $E W \mathrm{~s}$ (open squares). Lines represent predictions obtained analyzing with a constant microturbulent velocity synthetic spectra computed assuming parabolic runs of microturbulent velocity with optical depth, with minima at $\log \tau=0$ (solid line), $\log \tau=-1$ (dotted line), $\log \tau=-2$ (short dashed line), and $\log \tau=-3$ (long dashed line).

Figure 7. Departure coefficients for Fe I levels from our statistical equilibrium computations for a typical RR Lyrae model atmosphere $\left(T_{\text {eff }}=6200 \mathrm{~K}, \log g=2.75,[\mathrm{~A} / \mathrm{H}]=-1.3\right)$. Computations were made using MULTI code by Scharmer \& Carlsson (1985), and the 61-level Fe-I model atom by Gratton et al (1995). In panel (a) collisions with H I atoms are included $(k=31.6)$, while they are neglected in panel (b).

Figure 8. Calibration of the cross section for collisions with H I atoms from differences in the abundance derived from neutral and singly ionized Fe lines. The shaded region 
corresponds to the range allowed for these differences, by observational errors and uncertainties in the adopted atmospheric parameters.

Figure 9. Comparison of the $\alpha$-elements overabundance in RR Lyrae variables and non-variable less evolved stars in the solar neighborhood (Edvardsson et al 1993, Nissen et al 1994; Magain 1989; Zhao \& Magain 1990; Gratton \& Sneden 1991; Carretta et al 1995).

Figure 10(a-d). Comparison between synthetic and observed spectra for a few stars.

Figure 11. Calibration of the cross section for collisions with H I atoms from: a) the multiplets at 7771-7774 and 6155-58 $\AA$ of $\mathrm{O} \mathrm{I}$, and b) the doublets at 5893 and $5685 \AA$ of $\mathrm{Na}$ I. Shaded regions correspond to the range allowed by observational errors and uncertainties in the adopted atmospheric parameters.

Figure 12. Corrections to the LTE abundances of a) the O I IR triplet and b) the Na I lines, as a function of line strength, for model atmospheres representative of variables having different metallicities (solar, intermediate and low). Different curves in panel (b) correspond to the $5893 \AA$ doublet of $\mathrm{Na}$ I for $[\mathrm{Fe} / \mathrm{H}]=0.0$ (heavy solid line), $[\mathrm{Fe} / \mathrm{H}]=-1.5$ (heavy dotted line) and $[\mathrm{Fe} / \mathrm{H}]=-2.5$ (heavy short-dashed line), respectively; and the $5688 \AA$ doublet of $\mathrm{Na} \mathrm{I}$ for $[\mathrm{Fe} / \mathrm{H}]=0.0$ (light solid line), $[\mathrm{Fe} / \mathrm{H}]=-1.5$ (light dotted line) and $[\mathrm{Fe} / \mathrm{H}]=-2.5$ (light short-dashed line), respectively.

Figure 13. Comparison of the $[\mathrm{Na} / \mathrm{Mg}]$ abundances in RR Lyrae variables and non-variable less evolved stars in the solar neighborhood (Edvardsson et al 1993; Nissen et al 1994; Carretta et al 1995).

Figure 14. (a) The $\Delta \mathrm{S}$ vs $[\mathrm{Fe} / \mathrm{H}]$ calibration obtained from the stars analyzed in the present paper alone (solid line); (b) The $\Delta \mathrm{S}$ vs $[\mathrm{Fe} / \mathrm{H}]$ calibration obtained by combining our $[\mathrm{Fe} / \mathrm{H}]$ values (filled squares), and those by Butler and coworkers (open squares), corrected for the systematic difference with respect to our $[\mathrm{Fe} / \mathrm{H}]$ (see Section 5.1); (c) The $\Delta \mathrm{S}$ vs $[\mathrm{Fe} / \mathrm{H}]$ calibration obtained from the total sample of field (filled and open squares) and globular cluster RR Lyraes (asterisks), (see Section 5.1.1). This last one is our final recommended calibration.

Figure 15. The new $W^{\prime}(K)$ vs $[\mathrm{Fe} / \mathrm{H}]$ calibration (solid line), derived from stars in common with Clementini et al (1991). The dotted line represents the $W^{\prime}(K)$ vs $[\mathrm{Fe} / \mathrm{H}]$ calibration obtained in that paper.

Figure 16. $\mathrm{M}_{V}(\mathrm{RR})$ vs $[\mathrm{Fe} / \mathrm{H}]$ using the compilation in Table 21. Panel (a) corresponds to original $\mathrm{M}_{V}$ values (Column 5 of Table 21); panel (b) to $\mathrm{M}_{V}$ values corrected to $p=1.38$ (Column 6 of Table 21). Different symbols refer to: field RR Lyraes (open and filled squares, the last ones being the objects studied in the present paper, for them, we have used our 
[Fe/H] estimates); RR Lyraes in M5 and M92 (open triangles and open circles, respectively) according to Storm et al (1994); and RR Lyraes in M4 (filled triangles) according to Liu \& Janes (1991). Field variables that are claimed to be evolved are marked as asterisks. Best-fit regression lines obtained eliminating the evolved objects : SS Leo and M92 stars, are plotted on the data. 


\section{Table captions}

Table 1. Program stars and observation log

Table 2. Characteristics of the co-added spectra

Table 3a. List of lines and adopted gf values and their equivalent widths for : V445 Oph, SW And, V440 Sgr, RR Cet, UU Cet

Table 3b. List of lines and adopted gf values and their equivalent widths for : RR Lyr, VX Her, ST Boo, VY Ser, X Ari

Table 4. Reddening of program stars, from data in the literature

Table 5. Reddening estimates from interstellar absorption features

Table 6. Metallicity values from data in the literature

Table 7. Population I main sequence stars with accurate $T_{\text {eff }}$

Table 8. Effective temperatures

Table 9. $T_{\text {eff }}$ from $\mathrm{H}_{\alpha}$ profiles

Table 10. Adopted atmospheric parameters

Table 11. Dependence of abundances on atmospheric parameters

Table 12. Fe abundances

Table 13. Fe abundances from spectra at maximum light for SW And

Table 14. Abundances of $\alpha$-elements

Table 15. Overabundances of $\alpha$-elements in metal-poor RR Lyraestars $([\mathrm{Fe} / \mathrm{H}]<-1)$

Table 16. Abundances of Fe group-elements

Table 17. Abundances of heavy elements

Table 18. Overabundances of heavy elements in metal-poor RR Lyraestars $([\mathrm{Fe} / \mathrm{H}]<-1)$

Table 19. Average abundances for ionized species in metal-rich RR Lyrae

Table 20. Results for $\mathrm{C}, \mathrm{O}, \mathrm{Na}$ and $\mathrm{Al}$ abundances

Table 21. $\mathrm{M}_{V}(\mathrm{RR})$ from B-W 